\title{
Sensor Choice for Minimum Error Variance Estimation
}

\author{
Minxin Zhang and Kirsten Morris \\ Dept. of Applied Mathematics \\ Univ. of Waterloo \\ Waterloo, Canada \\ kmorriseuwaterloo.ca
}

\begin{abstract}
A Kalman filter is optimal in that the variance of the error is minimized by the estimator. It is shown here, in an infinite-dimensional context, that the solution to an operator Riccati equation minimizes the steady-state error variance. This extends a result previously known for lumped parameter systems to distributed parameter systems. It is shown then that minimizing the trace of the Riccati operator is a reasonable criterion for choosing sensor locations. It is then shown that multiple inaccurate sensors, that is, those with large noise variance, can provide as good an estimate as a single highly accurate (but probably more expensive) sensor. Optimal sensor location is then combined with estimator design. A framework for calculation of the best sensor locations using approximations is established and sensor location as well as choice is investigated with three examples. Simulations indicate that the sensor locations do affect the quality of the estimation and that multiple low quality sensors can lead to better estimation than a single high quality sensor.
\end{abstract}

\section{INTRODUCTION}

In most systems the full state is not measured, but must be estimated based on the available measurements. The accuracy of an estimate depends not only on the type of estimator, but also on the type of sensor. For distributed parameter systems, the location of the sensors is also a variable in estimator design. For a given expenditure, what is the best choice of sensors? What are the best locations?

The sensor selection and location problem has been considered by many researchers in various contexts. Observability is a common criterion for sensor placement. In [25], a method for optimal sensor placement based on observability on a thin double-curved shell structures was presented. The concept of partial observability was introduced in [18] to determine the optimal sensor locations in numerical weather predictions. Optimal actuator/sensor placement problem for transport-reaction processes, with respect to controllability and observability defined using spatial $\mathscr{H}_{2}$ norm, was considered in [1]. Optimizing the shape and the location of sensors with respect to observability was investigated in [22, 23, 24].

However, a common criterion for estimator design is to minimize the variance of the error. It makes sense to use the same criterion for sensor placement. This is reinforced by the results in [28] where it was shown that locations that maximize controllability do not generally minimize the linear

The financial support of a NSERC Discovery Grant and US AFOSR grant FA_9550-16-1-0061 for the research discussed in this article is gratefully acknowledged. quadratic cost for structural vibrations. Since minimum variance estimation is dual to optimal linear quadratic control and observability is dual to controllability, this suggests that points of maximum observability are not the best locations from the viewpoint of estimation error. Furthermore, there are numerical issues with maximizing controllability and observability for distributed parameter systems [28]. In [6], a procedure for sequential optimal sensor location for systems on a finite time interval was formulated, with the error variance at the final time as the optimality criterion. The procedure was applied to an example of a tubular-flow reactor system with multiple sensors placed both simultaneously and sequentially.

A Kalman filter is an optimal state estimator that minimizes the estimation error variance. The well-posedness of minimizing the error variance at a finite-time for distributed parameter systems was established in [3, 7]; see also the review [8] and the book [10]. Well-posedness of the timevarying case and conditions for using approximations in optimization of the sensor locations were established in [27] and applied to a problem of advection-diffusion. Conditions that ensure the existence of Bochner integrable solutions to infinitedimensional Riccati integral equations were established in [5], with an application to optimal sensor placement in a convection-diffusion example. In [4], the optimal filtering problem for mobile sensor networks was investigated, with the cost functional defined as the time integral of the trace of the weighted error covariance.

In many applications an infinite-time Kalman filter is used, partly because it leads to a constant operator that is simple to implement. In [17] the sensors were placed to minimize the trace of the Riccati matrix in a problem of channel flow estimation. In this paper, it is shown that the infinite-time Kalman filter minimizes the steady-state error variance for distributed parameter systems. This extends a result known for lumped parameter systems. More precisely, the steady-state error variance is shown to be the nuclear norm of the operator that solves an algebraic Riccati equation (ARE). This is used as the criterion for sensor placement. It is then proven that this cost decreases with the number of sensors and increases with sensor noise. Thus, if enough inaccurate sensors are used, the estimator error can be smaller than that obtained with a small number of accurate sensors. Sensor location that minimizes the error variance is the dual problem to the linear quadratic optimal actuator location. The results on well- 
posedness and also the use of approximations in calculating the optimal locations in [21] are used to obtain results on the computation of optimal sensor locations. In [13] an algorithm for computation of linear quadratic optimal actuator locations was presented and this is modified to use in computation of the optimal sensor locations here. The effect of the sensor noise and the number of sensors on the accuracy of an estimator is examined on a number of examples. A preliminary version of some of the results in this paper (without proofs) and the first example, were presented in [29].

\section{INFINITE-DIMENSIONAL KALMAN FILTER}

Consider an infinite-dimensional integral process

$$
z(t)=T(t) z_{0}+\int_{0}^{t} T(t-s) G d w(s),
$$

with measurement

$$
y(t)=\int_{0}^{t} C z(s) d s+v(t), t \geq 0,
$$

where $T(t)$ is a $C_{0}$-semigroup with infinitesimal generator $A$ on a separable Hilbert space $\mathscr{Z}, w(t)$ is a Wiener process of incremental covariance $Q$ on a separable Hilbert space $\mathscr{W}$. and $v(t)$ is a Wiener process of incremental covariance $R$ on a separable Hilbert space $\mathscr{Y}$. The operators $G$ and $C$ are bounded linear operators: $G \in \mathscr{L}(\mathscr{W}, \mathscr{Z})$, and $C \in \mathscr{L}(\mathscr{Z}, \mathscr{Y})$.

Definition 2.1: [10, Definition 5.2] For $\zeta_{1}, \zeta_{2} \in \mathscr{Z}$, define $\zeta_{1} \circ \zeta_{2} \in \mathscr{L}(\mathscr{Z})$ by

$$
\left(\zeta_{1} \circ \zeta_{2}\right) h=\zeta_{1}\left\langle\zeta_{2}, h\right\rangle, \text { for all } h \in \mathscr{Z} \text {. }
$$

The covariance operator of a $\mathscr{Z}$-valued random variable $\zeta$ with $E\left\{\|\zeta\|^{2}\right\}<\infty$ is defined by

$$
\operatorname{Cov}(\zeta)=E\{(\zeta-E \zeta) \circ(\zeta-E \zeta)\}
$$

Assume that the initial state $z_{0}$ is a $\mathscr{Z}$-valued Gaussian random variable, with zero mean value and covariance $P_{0}$. This implies that the nuclear norm

$$
\left\|P_{0}\right\|_{1}=E\left\{\left\|z_{0}\right\|^{2}\right\}<\infty
$$

([10, Page 118]). Also, assume the output space $\mathscr{Y}$ is finitedimensional, the operator $R \in \mathscr{L}(\mathscr{Y})$ is positive, and $w(t)$, $v(t), z_{0}$ are mutually uncorrelated. The inner product on $\mathscr{Z}$ will be indicated by $\langle\cdot, \cdot\rangle$ while the inner product on other Hilbert spaces will be specified by inclusion of a subscript, for instance $\langle\cdot, \cdot\rangle_{\mathscr{Y}}$.

Define

$$
\mathscr{B}_{2}([0, t] ; \mathscr{L}(\mathscr{Y}, \mathscr{Z}))=\left\{\begin{array}{cl}
\Phi: & \Phi(s) \in \mathscr{L}(\mathscr{Y}, \mathscr{Z}), s \in[0, t], \\
& \left\langle\Phi(\cdot) z_{1}, z_{2}\right\rangle \text { is measurable on } \\
& {[0, t] \text { for any } z_{1} \in \mathscr{Y}, z_{2} \in \mathscr{Z},} \\
& \text { and } \int_{0}^{t}\|\Phi(s)\|^{2} d t<\infty
\end{array}\right\} .
$$

The objective is to find an estimate $\tilde{z}(t)$ of the state $z(t)$ for each $t \geq 0$, based on the measured output $\{y(s): 0 \leq s \leq t\}$. More precisely, an estimate of the form

$$
\tilde{z}(t)=\int_{0}^{t} \Psi(t, s) d y(s),
$$

where $\Psi(t, \cdot) \in \mathscr{B}_{2}([0, t] ; \mathscr{L}(\mathscr{Y}, \mathscr{Z}))$ is sought.
Definition 2.2 (Mild evolution operator): [12, Def. 3.2.4, Def. 3.2.6] Let $\left[0, t_{1}\right]$ be a finite time interval, $\triangle\left(\left[0, t_{1}\right]\right)=$ $\left\{(t, s): t_{1} \geq t \geq s \geq 0\right\} \subset \mathbb{R}^{2}$, and

$\mathscr{B}_{\infty}\left(\left[0, t_{1}\right] ; \mathscr{L}(\mathscr{Z})\right)=\left\{\begin{array}{cl}D(t): & D(t) \in \mathscr{L}(\mathscr{Z}), \quad t \in\left[0, t_{1}\right], \\ & \left\langle D(t) z_{1}, z_{2}\right\rangle \text { is measurable on } \\ & {\left[0, t_{1}\right] \text { for any } z_{1}, z_{2} \in \mathscr{Z},} \\ & \text { and ess sup }\|D(t)\|<\infty\end{array}\right\}$.

(a) An operator $S(t, s): \triangle\left(\left[0, t_{1}\right]\right) \rightarrow \mathscr{L}(\mathscr{Z})$ is called a mild evolution operator if it has the following properties: (i) $S(s, s)=I$, for $s \in\left[0, t_{1}\right]$; (ii) $S(t, r) S(r, s)=S(t, s), 0 \leq s \leq$ $t \leq t_{1}$; (iii) For each $s \in\left[0, t_{1}\right], S(\cdot, s)$ is strongly continuous on $[s, \tau]$; (iv) For each $t \in\left[0, t_{1}\right], S(t, \cdot)$ is strongly continuous on $[0, t]$.

(b) Suppose $A$, with domain $\mathscr{D}(A)$, is the infinitesimal generator of the $C_{0}$-semigroup $T(t)$. For $D(t) \in \mathscr{B}_{\infty}\left(\left[0, t_{1}\right] ; \mathscr{L}(\mathscr{Z})\right)$ and arbitrary $z \in \mathscr{D}(A)$,

$S(t, s) z=T(t-s) z+\int_{s}^{t} T(t-\tau) D(\tau) S(\tau, s) z d \tau,(s, t) \in\left[0, t_{1}\right]$

has a unique solution in the class of mild evolution operators on $\mathscr{Z}$. The solution is called the mild evolution operator generated by $A+D(t)$.

Theorem 2.3 (Infinite-dimensional finite-time Kalman filter): [10, Theorem 6.9, Lemma 6.12] Let $\left[0, t_{1}\right]$ be a finite time interval, and $P(t) \in \mathscr{L}(\mathscr{Z})$ the unique self-adjoint, strongly continuous solution to the differential Riccati equation (DRE): for all $h_{1}, h_{2} \in \mathscr{D}\left(A^{*}\right)$,

$$
\begin{aligned}
\left\langle\dot{P}(t) h_{1}, h_{2}\right\rangle= & \left\langle\left[ A P(t)+P(t) A^{*}-P(t) C^{*} R^{-1} C P(t)\right.\right. \\
& \left.\left.+G Q G^{*}\right] h_{1}, h_{2}\right\rangle, \quad t \in\left[0, t_{1}\right], \\
P(0)= & P_{0} .
\end{aligned}
$$

Suppose $S_{p}(t, \cdot)$ is the mild evolution operator generated by $A-P(\cdot) C^{*} R^{-1} C$.

The estimate

$$
\tilde{z}_{o p t}(t)=\int_{0}^{t} S_{p}(t, s) P(s) C^{*} R^{-1} d y(s)
$$

is the unique optimal estimate for $z(t)$ in that for each $h \in \mathscr{Z}$,

$$
E\left\{\left\langle\left(z(t)-\tilde{z}_{o p t}(t), h\right\rangle^{2}\right\}=\min _{\tilde{z}} E\left\{\left\langle(z(t)-\tilde{z}(t), h\rangle^{2}\right\},\right.\right.
$$

where the minimum is taken over all estimates $\tilde{z}(t)$ of the form (3). The error covariance satisfies

$$
P(t)=E\left\{\left(z(t)-\tilde{z}_{o p t}(t)\right) \circ\left(z(t)-\tilde{z}_{o p t}(t)\right)\right\} .
$$

Moreover, letting $\|\cdot\|_{1}$ indicate the nuclear norm,

$$
\begin{aligned}
\|P(t)\|_{1} & =E\left\{\left\|z(t)-\tilde{z}_{o p t}(t)\right\|^{2}\right\} \\
& =\min _{\tilde{z}} E\left\{\|z(t)-\tilde{z}(t)\|^{2}\right\} .
\end{aligned}
$$

Definition 2.4: (a) A $C_{0}$-semigroup $T(t)$ on a Hilbert space $\mathscr{Z}$ is exponentially stable if there exist positive constants $M$ and $\alpha$ such that for $t \geq 0$

$$
\|T(t)\| \leq M e^{-\alpha t} .
$$

(b) The pair $(A, B)$ is exponentially stabilizable if there exists $K \in \mathscr{L}(\mathscr{Z}, \mathscr{X})$ such that $A-B K$ generates an exponentially stable $C_{0}$-semigroup. 
(c) The pair $(A, C)$ is exponentially detectable if there exists $F \in \mathscr{L}(\mathscr{Y}, \mathscr{Z})$ such that $A-F C$ generates an exponentially stable $C_{0}$-semigroup.

As time approaches infinity the Kalman filter converges to a time-invariant filter. The theorem below is presented in [10] and can be proven using [12, Theorems 6.2.4, 6.2.7] by duality.

Theorem 2.5: [10] If $(A, G \sqrt{Q})$ is exponentially stabilizable and $(A, C)$ is exponentially detectable, then the algebraic Riccati equation (ARE)

$$
\left\langle\left[A X+X A^{*}-X C^{*} R^{-1} C X+G Q G^{*}\right] h_{1}, h_{2}\right\rangle=0,
$$

for all $h_{1}, h_{2} \in \mathscr{D}\left(A^{*}\right)$, has a unique nonnegative solution, $P_{s s} \in$ $\mathscr{L}(\mathscr{Z})$, such that $A-P_{s s} C^{*} R^{-1} C$ generates an exponentially stable $C_{0}$-semigroup.

Furthermore, letting $P(t)$ indicate the solution to the differential Riccati equation (5), $P(t)$ converges strongly to $P_{s s}$, i.e. for all $h \in \mathscr{Z}$,

$$
\lim _{t \rightarrow \infty} P(t) h=P_{s s} h .
$$

Let $T_{p}(t)$ be the exponentially stable $C_{0}$-semigroup generated by $A-P_{s s} C^{*} R^{-1} C$ on $\mathscr{Z}$. The steady-state Kalman filter is characterized by

$$
\tilde{z}(t)=\int_{0}^{t} T_{p}(t-s) P_{s s} C^{*} R^{-1} d y(s) .
$$

It is now proven that $P(t)$ converges in nuclear norm to $P_{s s}$. The lemma below, needed in the proof of Theorem 2.7, is a special case of [15, Theorem 2].

Lemma 2.6: If a sequence of self-adjoint nuclear operators $X_{n} \in \mathscr{L}(\mathscr{Z})$ converges strongly to a nuclear operator $X \in$ $\mathscr{L}(\mathscr{Z})$, and $\left\|X_{n}\right\|_{1}$ converges to $\|X\|_{1}$, then

$$
\lim _{n \rightarrow \infty}\left\|X_{n}-X\right\|_{1}=0 .
$$

Theorem 2.7: Assume $(A, G \sqrt{Q})$ is exponentially stabilizable and $(A, C)$ is exponentially detectable. If both the spaces $\mathscr{W}$ and $\mathscr{Y}$ are finite-dimensional, then $P_{s s}$ is nuclear and

$$
\lim _{t \rightarrow \infty}\left\|P(t)-P_{s s}\right\|_{1}=0 .
$$

Furthermore,

$$
\begin{aligned}
\left\|P_{s s}\right\|_{1} & =\lim _{t \rightarrow \infty} E\left\{\left\|z(t)-\tilde{z}_{\text {opt }}(t)\right\|^{2}\right\} \\
& =\min _{\tilde{z}} \lim _{t \rightarrow \infty} E\left\{\|z(t)-\tilde{z}(t)\|^{2}\right\},
\end{aligned}
$$

where the minimum is taken over all estimates $\tilde{z}(t)$ of the form (3) such that the $\operatorname{limit} \lim _{t \rightarrow \infty} E\left\{\|z(t)-\tilde{z}(t)\|^{2}\right\}$ exists.

Proof: The fact that $P_{s s}$ is nuclear is a straightforward consequence of [9, Theorem 3.3] by duality and the assumption that both the input space for the process noise and measurement space $\mathscr{Y}$ are finite-dimensional.

Results on linear quadratic control [12, Chapter 6] will be used . Let $\mathscr{D}\left(A^{*}\right) \subset \mathscr{Z}$ be the domain of $A^{*}$. For arbitrary $h_{0} \in \mathscr{D}\left(A^{*}\right)$, consider the linear quadratic control problem for the dynamic system

$$
\dot{z}(t)=A^{*} z(t)+C^{*} u(t), \quad z(0)=h_{0}, \quad t \geq 0,
$$

where $u \in L_{2}([0,+\infty) ; \mathscr{Y})$ represents the control. By [12, Theorem 2.2.6], $A^{*}$ is the infinitesimal generator of the $C_{0^{-}}$ semigroup $T^{*}(t)$. Define the quadratic cost functionals

$$
\begin{aligned}
\tilde{J}\left(h_{0} ; 0, t, u\right):=\left\langle z(t), P_{0} z(t)\right\rangle & +\int_{0}^{t}\left(\left\langle z(s), G Q G^{*} z(s)\right\rangle\right. \\
& +\langle u(s), R u(s)\rangle) d s
\end{aligned}
$$

and

$$
\tilde{J}\left(h_{0} ; u\right):=\int_{0}^{\infty}\left\langle z(s), G Q G^{*} z(s)\right\rangle+\langle u(s), R u(s)\rangle d s .
$$

Applying [12, Theorem 6.1.13] to system (13), since $P(\cdot)$ is the unique solution of Riccati equation (5), for each $t \geq 0$,

$$
\left\langle P(t) h_{0}, h_{0}\right\rangle=\min _{u \in L_{2}([0, t] ; \mathscr{Y})} \tilde{J}\left(h_{0} ; 0, t, u\right) .
$$

Also, by the dual of [12, Theorem 6.2.4]

$$
\left\langle P_{s s} h_{0}, h_{0}\right\rangle=\min _{u \in L_{2}([0,+\infty) ; \mathscr{Y})} \tilde{J}\left(h_{0} ; u\right) .
$$

The minimum in (14) is achieved by using the state feedback control

$$
u_{s s}(t):=-R^{-1} C P_{s s} z(t),
$$

with the optimal state trajectory $z(t)=T_{p}^{*}(t) h_{0}$, in which $T_{p}^{*}(\cdot)$ is the exponentially stable $C_{0}$-semigroup on $\mathscr{Z}$ generated by $A^{*}-C^{*} R^{-1} C P_{s s}$. There exists positive constants $\alpha$ and $\beta$ such that

$$
\left\|T_{p}^{*}(t)\right\|=\left\|T_{p}(t)\right\| \leq \beta e^{-\alpha t}, \quad t \geq 0 .
$$

Thus

$$
\begin{aligned}
\left\langle P(t) h_{0}, h_{0}\right\rangle \leq & \tilde{J}\left(h_{0} ; 0, t, u_{s s}\right) \\
= & \left\langle T_{p}^{*}(t) h_{0}, P_{0} T_{p}^{*}(t) h_{0}\right\rangle+\int_{0}^{t}\left(\left\langle u_{s s}(s), R u_{s s}(s)\right\rangle\right. \\
& \left.+\left\langle T_{p}^{*}(s) h_{0}, G Q G^{*} T_{p}^{*}(s) h_{0}\right\rangle\right) d s \\
\leq & \left\langle T_{p}^{*}(t) h_{0}, P_{0} T_{p}^{*}(t) h_{0}\right\rangle+\tilde{J}\left(h_{0} ; u_{s s}\right) \\
= & \left\langle h_{0}, T_{p}(t) P_{0} T_{p}^{*}(t) h_{0}\right\rangle+\left\langle P_{s s} h_{0}, h_{0}\right\rangle
\end{aligned}
$$

for any $h_{0} \in \mathscr{D}\left(A^{*}\right)$. Since $A^{*}$ is the infinitesimal generator of the $C_{0}$-semigroup $T^{*}(\cdot)$, by [12, theorem 2.1.10.e], $\mathscr{D}\left(A^{*}\right)$ is dense in $\mathscr{Z}$. Hence for any $h \in \mathscr{Z}$,

$$
\langle P(t) h, h\rangle \leq\left\langle h, T_{p}(t) P_{0} T_{p}^{*}(t) h\right\rangle+\left\langle P_{s s} h, h\right\rangle .
$$

By [26, Theorem 7.8],

$$
\left\|T_{p}(t) P_{0} T_{p}^{*}(t)\right\|_{1} \leq\left\|T_{p}(t)\right\|^{2}\left\|P_{0}\right\|_{1},
$$

and since $T_{p}(t)$ is exponentially stable, there is $\alpha>0, \beta>0$ so that for all $t \geq 0$,

$$
\left\|T_{p}(t) P_{0} T_{p}^{*}(t)\right\|_{1} \leq \beta^{2} e^{-2 \alpha t}\left\|P_{0}\right\|_{1} .
$$

Let $\left\{\psi_{j}\right\}_{j=1}^{\infty}$ be an orthonormal basis of $\mathscr{Z}$. Combining (16) with (17), 


$$
\begin{aligned}
\|P(t)\|_{1}-\left\|P_{s s}\right\|_{1} & =\sum_{j=1}^{\infty}\left\langle\left(P(t)-P_{s s}\right) \psi_{j}, \psi_{j}\right\rangle \\
& \leq \sum_{j=1}^{\infty}\left\langle\psi_{j}, T_{p}(t) P_{0} T_{p}^{*}(t) \psi_{j}\right\rangle \\
& =\left\|T_{p}(t) P_{0} T_{p}^{*}(t)\right\|_{1} \\
& \leq\left\|T_{p}(t)\right\|^{2}\left\|P_{0}\right\|_{1} \\
& \leq \beta^{2} e^{-2 \alpha t}\left\|P_{0}\right\|_{1} .
\end{aligned}
$$

Thus, for any $\varepsilon>0$, there exists $t_{\varepsilon}>0$ such that for all $t>t_{\varepsilon}$,

$$
\|P(t)\|_{1}-\left\|P_{s s}\right\|_{1}<\varepsilon \text {. }
$$

On the other hand, since $P_{s s}$ is nuclear, there exists a positive integer $N_{\varepsilon}$ such that for any $N>N_{\mathcal{E}}$,

$$
\sum_{j=N}^{\infty}\left\langle P_{s s} \psi_{j}, \psi_{j}\right\rangle<\frac{\varepsilon}{2}
$$

Also, by Theorem 2.5, $P(t)$ converges strongly to $P_{s s}$. There exists $\tilde{t}_{\varepsilon}>0$ such that for any $t>\tilde{t}_{\varepsilon}$,

$$
\sum_{j=1}^{N_{\varepsilon}}\left|\left\langle\left(P_{s s}-P(t)\right) \psi_{j}, \psi_{j}\right\rangle\right|<\frac{\varepsilon}{2}
$$

It follows that for $t>\tilde{t}_{\varepsilon}$,

$$
\begin{aligned}
\left\|P_{s s}\right\|_{1}= & \sum_{j=1}^{N_{\varepsilon}}\left\langle\left(P_{s s}-P(t)\right) \psi_{j}, \psi_{j}\right\rangle+\sum_{j=1}^{N_{\varepsilon}}\left\langle P(t) \psi_{j}, \psi_{j}\right\rangle \\
& +\sum_{j=N_{\varepsilon}+1}^{\infty}\left\langle P_{s s} \psi_{j}, \psi_{j}\right\rangle \\
< & \frac{\varepsilon}{2}+\|P(t)\|_{1}+\frac{\varepsilon}{2}=\|P(t)\|_{1}+\varepsilon .
\end{aligned}
$$

Combining (18) and (19), for any $t>\max \left\{t_{\varepsilon}, \tilde{t}_{\varepsilon}\right\}$,

$$
\left\|P_{s s}\right\|_{1}-\varepsilon<\|P(t)\|_{1}<\left\|P_{s s}\right\|_{1}+\varepsilon \text {. }
$$

Since $\varepsilon>0$ is arbitrary,

$$
\lim _{t \rightarrow \infty}\|P(t)\|_{1}=\left\|P_{S s}\right\|_{1} .
$$

Moreover, since $P(t)$ converges strongly to $P_{s s}$, and the operators are self-adjoint, Lemma 2.6 then implies that

$$
\lim _{t \rightarrow \infty}\left\|P(t)-P_{s s}\right\|_{1}=0 .
$$

Hence $P(t)$ converges to $P_{s s}$ in nuclear norm.

Letting $t \rightarrow \infty$ in (8), by (20), there exists the limit

$$
\left\|P_{s s}\right\|_{1}=\lim _{t \rightarrow \infty} E\left\{\left\|z(t)-\tilde{z}_{o p t}(t)\right\|^{2}\right\} .
$$

Taking the minimum over all estimates $\tilde{z}(t)$ of the form (3) such that the $\operatorname{limit}_{\lim _{t \rightarrow \infty}} E\left\{\|z(t)-\tilde{z}(t)\|^{2}\right\}$ exists,

$$
\begin{aligned}
\left\|P_{s s}\right\|_{1} & =\lim _{t \rightarrow \infty} E\left\{\left\|z(t)-\tilde{z}_{\text {opt }}(t)\right\|^{2}\right\} \\
& \geq \min _{\tilde{z}} \lim _{t \rightarrow \infty} E\left\{\|z(t)-\tilde{z}(t)\|^{2}\right\} .
\end{aligned}
$$

On the other hand, assume $\tilde{z}(t)$ is an arbitrary estimate of the form (3) such that the $\operatorname{limit} \lim _{t \rightarrow \infty} E\left\{\|z(t)-\tilde{z}(t)\|^{2}\right\}$ exists. Since for any $t \geq 0$,

$$
E\left\{\left\|z(t)-\tilde{z}_{o p t}(t)\right\|^{2}\right\} \leq E\left\{\|z(t)-\tilde{z}(t)\|^{2}\right\},
$$

letting $t \rightarrow \infty$ in the above inequality,

$$
\lim _{t \rightarrow \infty} E\left\{\left\|z(t)-\tilde{z}_{\text {opt }}(t)\right\|^{2}\right\} \leq \lim _{t \rightarrow \infty} E\left\{\|z(t)-\tilde{z}(t)\|^{2}\right\} .
$$

Then by (21),

$$
\left\|P_{s s}\right\|_{1} \leq \lim _{t \rightarrow \infty} E\left\{\|z(t)-\tilde{z}(t)\|^{2}\right\} .
$$

Since $\tilde{z}(t)$ is arbitrary, combining (22) with (23), it follows that

$$
\left\|P_{S S}\right\|_{1}=\min _{\tilde{z}} \lim _{t \rightarrow \infty} E\left\{\|z(t)-\tilde{z}(t)\|^{2}\right\},
$$

where the minimum is taken over all estimates $\tilde{z}(t)$ of the form (3) such that the $\operatorname{limit}_{t \rightarrow \infty} E\left\{\|z(t)-\tilde{z}(t)\|^{2}\right\}$ exists.

\section{Sensor Choice}

Theorem 2.7 shows that $\left\|P_{s s}\right\|_{1}$ is the minimum steadystate estimate error variance. The value of $\left\|P_{s s}\right\|_{1}$ is dependent on the measurement operator $C$, and thus the number of sensors, as well as on the sensor noise covariance $R$. Selection and location of sensors that minimize $\left\|P_{s s}\right\|_{1}$ is therefore a reasonable design goal. In this section it is proven that sensors with smaller variance lead to a better estimate; that is the variance of the error estimate is reduced, and also that increasing the number of sensors reduces the error variance.

Consider sensing with observation operator $\tilde{C} \in \mathscr{L}(\mathscr{Z}, \mathscr{Y})$ such that the pair $(A, \tilde{C})$ is exponentially detectable, with noise covariance $\tilde{R} \in \mathscr{L}(\mathscr{Y})$ where $\tilde{R}$ is positive definite. Let $\tilde{P}_{s s}$ be the unique nonnegative solution to the ARE

$$
\left\langle\left[A X+X A^{*}-X \tilde{C}^{*} \tilde{R}^{-1} \tilde{C} X+G Q G^{*}\right] h_{1}, h_{2}\right\rangle=0,
$$

for all $h_{1}, h_{2} \in \mathscr{D}\left(A^{*}\right)$. The objective is to compare $\tilde{P}_{s s}$ to $P_{s s}$, the solution to (9) for estimation with the observation operator $C$ and noise covariance $R$. Define

$$
D=C^{*} R^{-1} C, \quad \tilde{D}=\tilde{C}^{*} \tilde{R}^{-1} \tilde{C} .
$$

In [11, Theorem 1.2] it was shown that if

$$
D \geq \tilde{D}
$$

then

$$
P_{s s} \leq \tilde{P}_{s s},
$$

which implies that

$$
\left\|P_{s s}\right\|_{1} \leq\left\|\tilde{P}_{s s}\right\|_{1} .
$$

In the next theorem, it is shown that a similar inequality holds for the finite-time Kalman filter, and this is extended to the infinite-time Kalman filter using Theorem 2.7. A similar approach was used in [3]. Here the proof is different, filling in some steps omitted in [3] and extending the result to the steady-state infinite-time situation.

Theorem 3.1: Assume the spaces $\mathscr{W}$ and $\mathscr{Y}$ are finitedimensional, $\tilde{C} \in \mathscr{L}(\mathscr{Z}, \mathscr{Y}), \tilde{R} \in \mathscr{L}(\mathscr{Y})$ is positive, the pair $(A, G \sqrt{Q})$ is exponentially stabilizable, $(A, C)$ and $(A, \tilde{C})$ are exponentially detectable. Let $P(t)$ be the unique solution to the DRE (5) and $\tilde{P}(t)$ is the unique solution to the DRE with $C$ replaced by $\tilde{C}$ and $R$ replaced by $\tilde{R}$. Defining $D=C^{*} R^{-1} C$, and $\tilde{D}=\tilde{C}^{*} \tilde{R}^{-1} \tilde{C}$, if

$$
D \geq \tilde{D}
$$


then for any $t_{1}>0$,

$$
\left\|P\left(t_{1}\right)\right\|_{1} \leq\left\|\tilde{P}\left(t_{1}\right)\right\|_{1} .
$$

Furthermore, letting $P_{S S}$ be the unique nonnegative solution to the ARE (9) and $\tilde{P}_{s s}$ the non-negative solution to the corresponding ARE (24),

$$
\left\|P_{S S}\right\|_{1} \leq\left\|\tilde{P}_{S S}\right\|_{1}
$$

Proof: First, consider an arbitrary finite time interval $\left[0, t_{1}\right]$. By Theorem 2.3, for all $h_{1}, h_{2} \in \mathscr{D}\left(A^{*}\right)$,

$$
\begin{aligned}
\left\langle\dot{\tilde{P}}(t) h_{1}, h_{2}\right\rangle= & \left\langle\left[ A \tilde{P}(t)+\tilde{P}(t) A^{*}-\tilde{P}(t) \tilde{D} \tilde{P}(t)\right.\right. \\
& \left.\left.+G Q G^{*}\right] h_{1}, h_{2}\right\rangle \\
\tilde{P}(0)= & P_{0} .
\end{aligned}
$$

For an arbitrary $h \in \mathscr{Z}$, let $\phi(t)$ and $\tilde{\phi}(t)$ solve the equations

$$
\dot{\phi}(t)=\left(-A^{*}+D P(t)\right) \phi(t), \phi\left(t_{1}\right)=h, 0 \leq t \leq t_{1},
$$

and

$$
\dot{\tilde{\phi}}(t)=\left(-A^{*}+\tilde{D} \tilde{P}(t)\right) \tilde{\phi}(t), \tilde{\phi}\left(t_{1}\right)=h, 0 \leq t \leq t_{1}
$$

respectively. Define

$$
\varphi(t):=P(t) \phi(t), \quad \tilde{\varphi}(t):=\tilde{P}(t) \tilde{\phi}(t) .
$$

For $0 \leq t \leq t_{1}$, there exists the weak derivative

$$
\begin{aligned}
\dot{\varphi}(t)= & \dot{P}(t) \phi(t)+P(t) \dot{\phi}(t) \\
= & {\left[A P(t)+P(t) A^{*}-P(t) D P(t)+G Q G^{*}\right] \phi(t) } \\
& +P(t)\left(-A^{*}+D P(t)\right) \phi(t) \\
= & A \varphi(t)+G Q G^{*} \phi(t) .
\end{aligned}
$$

Similarly,

$$
\dot{\tilde{\varphi}}(t)=A \tilde{\varphi}(t)+G Q G^{*} \tilde{\phi}(t), 0 \leq t \leq t_{1},
$$

in the weak sense. Define

$$
\theta(t):=\phi(t)-\tilde{\phi}(t), \quad \gamma(t):=\varphi(t)-\tilde{\varphi}(t) .
$$

It follows that

$$
\dot{\gamma}(t)=A \gamma(t)+G Q G^{*} \theta(t), \gamma(0)=P_{0} \theta(0), 0 \leq t \leq t_{1},
$$

and

$$
\begin{aligned}
& \dot{\theta}(t)=-A^{*} \theta(t)+D \gamma(t)+(D-\tilde{D}) \tilde{\varphi}(t), \theta\left(t_{1}\right)=0, \\
& 0 \leq t \leq t_{1}
\end{aligned}
$$

in the weak sense. Thus,

$$
\begin{aligned}
& \int_{0}^{t_{1}}\langle(D-\tilde{D}) \tilde{\varphi}(t), \tilde{\varphi}(t)\rangle d t \\
= & \int_{0}^{t_{1}}\left\langle\dot{\theta}(t)+A^{*} \theta(t), \tilde{\varphi}(t)\right\rangle d t-\int_{0}^{t_{1}}\langle D \gamma(t), \tilde{\varphi}(t)\rangle d t .
\end{aligned}
$$

Note that

$$
\begin{aligned}
\int_{0}^{t_{1}}\langle\dot{\theta}(t), \tilde{\varphi}(t)\rangle d t & =\int_{0}^{t_{1}} \frac{d}{d t}[\langle\theta(t), \tilde{\varphi}(t)\rangle] d t-\int_{0}^{t_{1}}\langle\theta(t), \dot{\tilde{\varphi}}(t)\rangle d t \\
& =-\langle\theta(0), \tilde{\varphi}(0)\rangle-\int_{0}^{t_{1}}\langle\theta(t), \dot{\tilde{\varphi}}(t)\rangle d t .
\end{aligned}
$$

Substituting into (29),

$$
\begin{aligned}
& \int_{0}^{t_{1}}\langle(D-\tilde{D}) \tilde{\varphi}(t), \tilde{\varphi}(t)\rangle d t \\
= & -\langle\theta(0), \tilde{\varphi}(0)\rangle-\int_{0}^{t_{1}}\langle\theta(t), \dot{\tilde{\varphi}}(t)\rangle d t+\int_{0}^{t_{1}}\langle\theta(t), A \tilde{\varphi}(t)\rangle d t \\
& -\int_{0}^{t_{1}}\langle D \gamma(t), \tilde{\varphi}(t)\rangle d t \\
= & -\langle\theta(0), \tilde{\varphi}(0)\rangle-\int_{0}^{t_{1}}\left(\left\langle\theta(t), G Q G^{*} \tilde{\phi}(t)\right\rangle+\langle D \gamma(t), \tilde{\varphi}(t)\rangle\right) d t \\
= & -\langle\theta(0), \tilde{\varphi}(0)\rangle-\int_{0}^{t_{1}}(\langle\dot{\gamma}(t)-A \gamma(t), \tilde{\phi}(t)\rangle+\langle D \gamma(t), \tilde{\varphi}(t)\rangle) d t .
\end{aligned}
$$

Also,

$$
\begin{aligned}
& \int_{0}^{t_{1}}\langle\dot{\gamma}(t), \tilde{\phi}(t)\rangle d t \\
= & \int_{0}^{t_{1}} \frac{d}{d t}[(\langle\gamma(t), \tilde{\phi}(t)\rangle)] d t-\int_{0}^{t_{1}}\langle\gamma(t), \dot{\tilde{\phi}}(t)\rangle d t \\
= & \left\langle\gamma\left(t_{1}\right), h\right\rangle-\langle\gamma(0), \tilde{\phi}(0)\rangle-\int_{0}^{t_{1}}\langle\gamma(t), \dot{\tilde{\phi}}(t)\rangle d t .
\end{aligned}
$$

Since

$$
\langle\theta(0), \tilde{\varphi}(0)\rangle=\left\langle\theta(0), P_{0} \tilde{\phi}(0)\right\rangle=\langle\gamma(0), \tilde{\phi}(0)\rangle,
$$

it follows that

$$
\begin{aligned}
& \int_{0}^{t_{1}}\langle(D-\tilde{D}) \tilde{\varphi}(t), \tilde{\varphi}(t)\rangle d t \\
= & -\left\langle\gamma\left(t_{1}\right), h\right\rangle+\int_{0}^{t_{1}}\langle\gamma(t), \dot{\tilde{\phi}}(t)\rangle d t+\int_{0}^{t_{1}}\langle A \gamma(t), \tilde{\phi}(t)\rangle d t \\
& -\int_{0}^{t_{1}}\langle D \gamma(t), \tilde{\varphi}(t)\rangle d t \\
= & -\left\langle\gamma\left(t_{1}\right), h\right\rangle+\int_{0}^{t_{1}}\left\langle\gamma(t), \dot{\tilde{\phi}}(t)+A^{*} \tilde{\phi}(t)-D \tilde{\varphi}(t)\right\rangle d t \\
= & -\left\langle\gamma\left(t_{1}\right), h\right\rangle+\int_{0}^{t_{1}}\langle\gamma(t),(\tilde{D}-D) \tilde{\varphi}(t)\rangle d t
\end{aligned}
$$

Also, by (27) and (28),

$$
\begin{aligned}
& \int_{0}^{t_{1}}\langle\gamma(t),(\tilde{D}-D) \tilde{\varphi}(t)\rangle d t \\
= & \int_{0}^{t_{1}}\left\langle\gamma(t), \dot{\theta}(t)+A^{*} \theta(t)-D \gamma(t)\right\rangle d t \\
= & -\langle\gamma(0), \theta(0)\rangle-\int_{0}^{t_{1}}\langle\dot{\gamma}(t), \theta(t)\rangle d t+\int_{0}^{t_{1}}\langle A \gamma(t), \theta(t)\rangle d t \\
& -\int_{0}^{t_{1}}\langle\gamma(t), D \gamma(t)\rangle d t \\
= & -\left\langle P_{0} \theta(0), \theta(0)\right\rangle-\int_{0}^{t_{1}}\left\langle G Q^{*} G \theta(t), \theta(t)\right\rangle d t-\int_{0}^{t_{1}}\langle\gamma(t), D \gamma(t)\rangle d t .
\end{aligned}
$$

Hence,

$$
\begin{aligned}
& \int_{0}^{t_{1}}\langle(D-\tilde{D}) \tilde{\varphi}(t), \tilde{\varphi}(t)\rangle d t \\
= & -\left\langle\gamma\left(t_{1}\right), h\right\rangle-\left\langle P_{0} \theta(0), \theta(0)\right\rangle-\int_{0}^{t_{1}}\left\langle G Q^{*} G \theta(t), \theta(t)\right\rangle d t \\
& -\int_{0}^{t_{1}}\langle\gamma(t), D \gamma(t)\rangle d t .
\end{aligned}
$$

Notice that

$\gamma\left(t_{1}\right)=\varphi\left(t_{1}\right)-\tilde{\varphi}\left(t_{1}\right)=P\left(t_{1}\right) \phi\left(t_{1}\right)-\tilde{P}\left(t_{1}\right) \tilde{\phi}\left(t_{1}\right)=\left(P\left(t_{1}\right)-\tilde{P}\left(t_{1}\right)\right) h$. 
Therefore,

$$
\begin{aligned}
& \left\langle\left(\tilde{P}\left(t_{1}\right)-P\left(t_{1}\right)\right) h, h\right\rangle \\
= & \left\langle-\gamma\left(t_{1}\right), h\right\rangle \\
= & \int_{0}^{t_{1}}\langle(D-\tilde{D}) \tilde{\varphi}(t), \tilde{\varphi}(t)\rangle d t+\left\langle P_{0} \theta(0), \theta(0)\right\rangle \\
& +\int_{0}^{t_{1}}\left(\left\langle G Q G^{*} \theta(t), \theta(t)\right\rangle+\langle\gamma(t), D \gamma(t)\rangle\right) d t .
\end{aligned}
$$

If

$$
D \geq \tilde{D},
$$

then, since $P_{0}, G Q G^{*}, D$ are nonnegative operators,

$$
\left\langle\left(\tilde{P}\left(t_{1}\right)-P\left(t_{1}\right)\right) h, h\right\rangle \geq 0 .
$$

Since $h$ is arbitrary, for any orthonormal basis of $\mathscr{Z},\left\{\psi_{j}\right\}_{j=1}^{\infty}$,

$$
\left\|\tilde{P}\left(t_{1}\right)\right\|_{1}-\left\|P\left(t_{1}\right)\right\|_{1}=\sum_{j=1}^{\infty}\left\langle\left(\tilde{P}\left(t_{1}\right)-P\left(t_{1}\right)\right) \psi_{j}, \psi_{j}\right\rangle \geq 0 .
$$

Hence, for any $t_{1} \geq 0$,

$$
\left\|P\left(t_{1}\right)\right\|_{1} \leq\left\|\tilde{P}\left(t_{1}\right)\right\|_{1} .
$$

It then follows from Theorem 2.7 that

$$
\left\|P_{s s}\right\|_{1} \leq\left\|\tilde{P}_{s s}\right\|_{1} .
$$

Now suppose that $m$ sensors are used. The measurement operator $C \in \mathscr{L}\left(\mathscr{Z}, \mathbb{R}^{m}\right)$ can be written as

$$
C=(C(1), C(2), \ldots, C(m))^{T},
$$

where $C(j) \in \mathscr{L}(\mathscr{Z}, \mathbb{R}), j=1,2, \ldots, m$, represents the measurement operator of the $j$-th sensor. Let $v_{j}(t)(j=1,2, \ldots, m)$ represent the noise in the $j$-th sensor. Assume the noises $v_{1}(t), v_{2}(t), \ldots, v_{m}(t)$ are mutually independent real-valued Wiener processes, with

$$
E\left\{\left(v_{j}(t)-v_{j}(s)\right)^{2}\right\}=(t-s) r_{j}, j=1,2, \ldots, m
$$

for $t \geq s \geq 0$, where $r_{j} \in \mathbb{R}_{+}$. If the sensor is of high quality, then $r_{j} \in \mathbb{R}_{+}$is small, while $r_{j}$ is larger for a low-quality sensor. If the $m$ sensors each have identical variance $r_{0}$ then the covariance of the measurement noise $v(t)$ is

$$
R=\operatorname{diag}\left(r_{0}, r_{0} \ldots r_{0}\right)=r_{0} I_{m},
$$

where $I_{m}$ represents the $m$-dimensional identity matrix.

The following result is now a straightforward consequence of Theorem 3.1.

Theorem 3.2: Let $P_{s s}$ be the Riccati operator that solves (9) for $m$ identical sensors with variance $r_{0}$, and similarly let $\tilde{P}_{s s}$ be the operator obtained for $m$ sensors with variance $\tilde{r}_{0}$. If $\tilde{r}_{0} \leq r_{0}$ then

$$
\left\|\tilde{P}_{s s}\right\|_{1} \leq\left\|P_{s s}\right\|_{1} .
$$

Also, comparing the performance of identical sensors, if $m_{1}>m$ sensors are used then the estimate is improved. Letting $\tilde{P}_{s s}$ be the Riccati operator with $m_{1}$ sensors, and $P_{s s}$ the Riccati operator with $m$ sensors, then

$$
\left\|\tilde{P}_{s s}\right\|_{1} \leq\left\|P_{s s}\right\|_{1} .
$$

Proof: If $\tilde{r}_{0} \leq r_{0}$, then

$$
C^{*} \tilde{R}^{-1} C=\tilde{r}_{0}^{-1} C^{*} C \geq r_{0}^{-1} C^{*} C=C^{*} R^{-1} C .
$$

By Theorem 3.1,

$$
\left\|\tilde{P}_{s s}\right\|_{1} \leq\left\|P_{s s}\right\|_{1} .
$$

Now suppose that the observation is obtained by $m$ identical sensors each with variance $r_{0}$

$$
C=[C(1), C(2), \ldots, C(m)],
$$

or $m_{1}>m$ of the same sensors:

$$
\tilde{C}=\left[C(1), C(2), \ldots, C(m), \ldots, C\left(m_{1}\right)\right] .
$$

Then,

$$
\left\langle\tilde{C}^{*} \tilde{C} h, h\right\rangle=\sum_{j=1}^{m_{1}}\|C(j) h\|^{2} \geq \sum_{j=1}^{m}\|C(j) h\|^{2}=\left\langle C^{*} C h, h\right\rangle .
$$

Thus,

$$
\tilde{C}^{*} \tilde{C} \geq C^{*} C
$$

and

$$
\tilde{C}^{*} \tilde{R}^{-1} \tilde{C}=r_{0}^{-1} \tilde{C}^{*} \tilde{C} \geq r_{0}^{-1} C^{*} C=C^{*} R^{-1} C .
$$

Again using Theorem 3.1,

$$
\left\|\tilde{P}_{s S}\right\|_{1} \leq\left\|P_{s s}\right\|_{1} .
$$

\section{OPTIMAL SENSOR LOCATION}

Suppose $m$ sensors with corresponding measurements

$$
y(t)=\left(y_{1}(t), y_{2}(t), \ldots, y_{m}(t)\right)^{T} \in \mathbb{R}^{m}, \quad t \geq 0
$$

are available. The sensors lie within some compact set $\Omega \subset \mathbb{R}^{q}$. Generally in applications $\Omega$ is a region in space so $q \leq 3$. Denoting the location of the $m$ sensors by

$$
\boldsymbol{l}:=\left(l_{1}, l_{2}, \ldots, l_{m}\right) \in \Omega^{m} \subset \mathbb{R}^{q \times m} ;
$$

the output operator $C$ is parameterized by the sensor location:

$$
C=C(l) .
$$

The location of the sensors should be chosen to minimize the error variance.

Continuity of $\left\|P_{s s}\right\|_{1}$ with respect to the sensor location $l$ follows from [21, Theorem 2.10] by duality.

Theorem 4.1: Assume $\mathscr{W}$ and $\mathscr{Y}$ are finite-dimensional. Let $C(\boldsymbol{l}) \in \mathscr{L}(\mathscr{Z}, \mathscr{Y}), \boldsymbol{l} \in \Omega^{m}$, be a family of output operators such that for any $l_{0} \in \Omega^{m}$,

$$
\left.\lim _{\boldsymbol{l} \rightarrow \boldsymbol{l}_{0}} \| C(\boldsymbol{l})-C\left(\boldsymbol{l}_{0}\right)\right) \|=0 .
$$

If $(A, G \sqrt{Q})$ is exponentially stabilizable and $(A, C(l))$ is exponentially detectable, then the corresponding Riccati operators $P_{s s}=P_{s s}(\boldsymbol{l})$ are continuous with respect to $\boldsymbol{l}$ in the nuclear norm:

$$
\lim _{l \rightarrow l_{0}}\left\|P_{s s}(\boldsymbol{l})-P_{s s}\left(\boldsymbol{l}_{0}\right)\right\|_{1}=0,
$$

and there exists an optimal sensor location $\hat{l}$ such that

$$
\left\|P_{s s}(\hat{\boldsymbol{l}})\right\|_{1}=\min _{l \in \Omega^{m}}\left\|P_{s s}(\boldsymbol{l})\right\|_{1} .
$$


For an infinite-dimensional system, the solution $P_{s s}$ to the ARE (9) cannot be computed exactly. Commonly, the system is approximated by a finite-dimensional system and the corresponding finite-dimensional ARE is solved. For $n \geq 1$, let $\mathscr{Z}_{n}$ be an $n$-dimensional subspace of $\mathscr{Z}$ with inner product inherited from $\mathscr{Z}$ and $\Pi_{n} \in \mathscr{L}\left(\mathscr{Z}, \mathscr{Z}_{n}\right)$ the orthogonal projection of $\mathscr{Z}$ onto $\mathscr{Z}_{n}$. Denote the original system (1) by $(A, C, G)$. Let $z_{n}=\Pi_{n}(z) \in \mathscr{Z}_{n}$ and approximate the system $(A, C, G)$ by a sequence of $\left(A_{n}, C_{n}, G_{n}\right)$, with $A_{n} \in \mathscr{L}\left(\mathscr{Z}_{n}, \mathscr{Z}_{n}\right)$, $C_{n}=\left.C\right|_{\mathscr{Z}_{n}} \in \mathscr{L}\left(\mathscr{Z}_{n}, \mathscr{Y}\right)$ (the restriction of $C$ to $\mathscr{Z}_{n}$ ), and $G_{n}=\Pi_{n} G \in \mathscr{L}\left(\mathscr{W}, \mathscr{Z}_{n}\right)$. Since $\mathscr{Z}_{n}$ is also a Hilbert space, the previous theorems in this paper apply to the approximate system. If $\left(A_{n}, C_{n}\right)$ is exponentially detectable and $\left(A_{n}, G_{n} \sqrt{Q}\right)$ is exponentially stabilizable, then by Theorem 2.5 , the finitedimensional ARE

$$
A_{n} X+X A_{n}^{*}-X C_{n}^{*} R^{-1} C_{n} X+G_{n} Q G_{n}^{*}=0,
$$

has a unique nonnegative solution $P_{s s}^{(n)} \in \mathscr{L}\left(\mathscr{Z}_{n}\right)$. The existence of an optimal sensor location vector $\hat{l}_{n}$ that minimizes the value of $\left\|P_{S S}^{(n)}\right\|_{1}$ is guaranteed by Theorem 4.1.

Conditions that ensure that the calculated optimal filter and sensor locations converge to the exact optimal filter and sensor locations as the approximation order is increased are needed. Let $T_{n}(t)$ indicate the semigroup of operators generated by $A_{n}$. The standard assumptions for using approximations in controller (and filter) design are as follows:

(H1) For each $z \in \mathscr{Z}$,

(i) $\left\|T_{n}(t) \Pi_{n} z-T(t) z\right\| \rightarrow 0$

(ii) $\left\|T_{n}^{*}(t) \Pi_{n} z-T^{*}(t) z\right\| \rightarrow 0$

uniformly in $t$ on bounded intervals.

(H2) For each $z \in \mathscr{Z}, w \in \mathscr{W}, y \in \mathscr{Y}$,

(i) $\left\|G_{n} w-G w\right\| \rightarrow 0$ and $\left\|C_{n} \Pi_{n} z-C z\right\| \rightarrow 0$,

(ii) $\left\|G_{n}^{*} \Pi_{n} z-G^{*} z\right\| \rightarrow 0$ and $\left\|C_{n}^{*} y-C^{*} y\right\| \rightarrow 0$.

(H3) (i) The family of pairs $\left(A_{n}, G_{n} \sqrt{Q}\right)$ is uniformly exponentially stabilizable, that is, there exists a uniformly bounded sequence of operators $K_{n} \in$ $\mathscr{L}\left(\mathscr{Z}_{n}, \mathscr{W}\right)$ such that the semigroups $T_{K_{n}}(t)$ generated by $A_{n}-G_{n} \sqrt{Q} K_{n}$ satisfy

$$
\left\|T_{K_{n}}(t)\right\| \leq \beta_{1} e^{-\alpha_{1} t}
$$

for positive constants $\alpha_{1}$ and $\beta_{1} \geq 1$.

(ii) The family of pairs $\left(A_{n}, C_{n}\right)$ is uniformly exponentially detectable, that is, there exists a uniformly bounded sequence of operators $F_{n} \in \mathscr{L}\left(\mathscr{Y}, \mathscr{Z}_{n}\right)$ such that the semigroups $T_{F_{n}}(t)$ generated by $A_{n}-$ $F_{n} C_{n}$ satisfy

$$
\left\|T_{F_{n}}(t)\right\| \leq \beta_{2} e^{-\alpha_{2} t}
$$

for positive constants $\alpha_{2}$ and $\beta_{2} \geq 1$.

These assumptions guarantee the convergence of $P_{s s}^{(n)}$ to the infinite-dimensional Riccati operator $P_{s s}$ in nuclear norm. The following result follows from [21, Theorem 3.8] by duality.

Theorem 4.2: Assume that $(A, G \sqrt{Q})$ is exponentially stabilizable, $(A, C)$ is exponentially detectable, and the spaces $\mathscr{W}$ and $\mathscr{Y}$ are finite-dimensional. Let $\left(A_{n}, G_{n}, C_{n}\right)$ be a sequence of approximations to $(A, G, C)$ that satisfies assumptions (H1)(H3) . Then

$$
\lim _{n \rightarrow \infty}\left\|P_{s s}^{(n)} \Pi_{n}-P_{s s}\right\|_{1}=0,
$$

where $\Pi_{n}$ is the orthogonal projection of $\mathscr{Z}$ onto $\mathscr{Z}_{n}$.

With the convergence (31), optimal sensor locations for approximate systems also converge to the optimal sensor location for the infinite-dimensional system. The following result is a dual version of [21, Theorem 3.9], and the proof follows that of [21, Theorem 3.5].

Theorem 4.3: Let $C(\boldsymbol{l}) \in \mathscr{L}(\mathscr{Z}, \mathscr{Y}), \boldsymbol{l} \in \Omega^{m}$, be a family of output operators such that for any $l_{0} \in \Omega^{m}$,

$$
\lim _{\boldsymbol{l} \rightarrow l_{0}}\left\|C(\boldsymbol{l})-C\left(\boldsymbol{l}_{0}\right)\right\|=0 .
$$

Assume that $(A, G \sqrt{Q})$ is exponentially stabilizable, $(A, C(\boldsymbol{l}))$, $\boldsymbol{l} \in \Omega^{m}$, are all exponentially detectable, and for each $\boldsymbol{l} \in$ $\Omega^{m},\left\{\left(A_{n}, C_{n}(\boldsymbol{l}), G_{n}\right)\right\}_{n=1}^{\infty}$ is a sequence of approximations for $(A, C(l)), G)$ that satisfies assumptions (H1)-(H3), with $C_{n}(\boldsymbol{l})=\left.C(\boldsymbol{l})\right|_{\mathscr{Z}_{n}}$. Letting $\hat{l}$ be an optimal sensor location with

$$
\left\|P_{S S}(\hat{\boldsymbol{l}})\right\|_{1}=\min _{l \in \Omega^{m}}\left\|P_{S S}(\boldsymbol{l})\right\|_{1} .
$$

and defining similarly $\hat{l}_{n}$,

$$
\left\|P_{s s}(\hat{\boldsymbol{l}})\right\|_{1}=\lim _{n \rightarrow \infty}\left\|P_{s s}^{(n)}\left(\hat{\boldsymbol{l}}_{n}\right)\right\|_{1},
$$

and there exists a subsequence $\left\{\hat{l}_{n_{k}}\right\}_{k=1}^{\infty}$ of $\left\{\hat{\boldsymbol{l}}_{n}\right\}_{n=1}^{\infty}$ such that $\lim _{k \rightarrow \infty} \hat{l}_{n_{k}}=\hat{\boldsymbol{l}}$. Moreover, any convergent subsequence of $\left\{\hat{l}_{n}\right\}_{n=1}^{\infty}$ converges to an optimal sensor location.

Proof: Write

$$
\hat{\mu}_{n}=\left\|P_{S S}^{(n)}\left(\hat{\boldsymbol{l}}_{n}\right)\right\|_{1}
$$

and

$$
\hat{\mu}=\left\|P_{s s}(\hat{\boldsymbol{l}})\right\|_{1}
$$

Then

$$
\begin{aligned}
\hat{\mu}_{n} & \leq\left\|P_{s s}^{(n)}(\hat{\boldsymbol{l}})\right\|_{1} \\
& \leq\left\|P_{s s}^{(n)}(\hat{\boldsymbol{l}}) \Pi_{n}-P_{s s}(\hat{\boldsymbol{l}})\right\|_{1}+\left\|P_{s s}(\hat{\boldsymbol{l}})\right\|_{1} .
\end{aligned}
$$

Therefore, by Theorem 4.2,

$$
\limsup _{n \rightarrow \infty} \hat{\mu}_{n} \leq \hat{\mu} .
$$

It will now be shown that

$$
\liminf _{n \rightarrow \infty} \hat{\mu}_{n} \geq \hat{\mu} .
$$

Let $\left\{\hat{\mu}_{n_{k}}\right\}_{k=1}^{\infty}$ be a subsequence of $\left\{\hat{\mu}_{n}\right\}_{n=1}^{\infty}$ such that

$$
\lim _{k \rightarrow \infty} \hat{\mu}_{n_{k}}=\liminf _{n \rightarrow \infty} \hat{\mu}_{n}
$$

Since $\Omega^{m}$ is compact, there exists a convergent subsequence of $\left\{\hat{\boldsymbol{l}}_{n_{k}}\right\}_{k=1}^{\infty} \subset \Omega^{m}$, also denoted by $\left\{\hat{\boldsymbol{l}}_{n_{k}}\right\}_{k=1}^{\infty}$ for simplicity, with limit $\underline{l}$. By assumption (H3)(ii), there exists a sequence of operators $F_{n}(\underline{l}) \in \mathscr{L}\left(\mathscr{Y}, \mathscr{Z}_{n}\right)$ such that for any $n \geq 1$,

$$
\left\|F_{n}(\underline{l})\right\| \leq \beta_{0}
$$

for some positive constant $\beta_{0}$, and the semigroups $T_{F_{n}(\underline{l})}(t)$ generated by $A_{n}-F_{n}(\underline{l}) C_{n}(\underline{l})$ satisfy

$$
\left\|T_{F_{n}(\underline{l})}(t)\right\| \leq \beta_{2} e^{-\alpha_{2} t}
$$


for some positive constants $\alpha_{2}$ and $\beta_{2} \geq 1$. Also,

$$
\lim _{k \rightarrow \infty}\left\|C_{n_{k}}\left(\hat{\boldsymbol{l}}_{n_{k}}\right)-C_{n_{k}}(\underline{\boldsymbol{l}})\right\| \leq \lim _{k \rightarrow \infty}\left\|C\left(\hat{\boldsymbol{l}}_{n_{k}}\right)-C(\underline{\boldsymbol{l}})\right\|=0 .
$$

For any positive constant $\varepsilon<\frac{\alpha_{2}}{\beta_{0} \beta_{2}}$, there exists $N_{\varepsilon}$ such that for $k \geq N_{\varepsilon}$,

$$
\left\|C_{n_{k}}\left(\hat{\boldsymbol{l}}_{n_{k}}\right)-C_{n_{k}}(\underline{l})\right\| \leq \varepsilon .
$$

Then by [12, Theorem 3.2.1], $A_{n_{k}}-F_{n_{k}}(\underline{\boldsymbol{l}}) C_{n_{k}}\left(\hat{\boldsymbol{l}}_{n_{k}}\right)$ generates a semigroup $T_{F_{n}\left(\hat{l}_{n_{k}}\right)}(t)$ such that

$$
\left\|T_{F_{n}\left(\hat{\boldsymbol{l}}_{n_{k}}\right)}(t)\right\| \leq \beta_{2} e^{\left(-\alpha_{2}+\beta_{0} \beta_{2} \varepsilon\right) t}
$$

for $k \geq N$. Moreover,

$$
\begin{aligned}
& \left\|C_{n_{k}}\left(\hat{\boldsymbol{l}}_{n_{k}}\right) \Pi_{n_{k}}-C(\underline{\boldsymbol{l}})\right\|=\left\|C\left(\hat{\boldsymbol{l}}_{n_{k}}\right) \Pi_{n_{k}}-C(\underline{\boldsymbol{l}})\right\| \\
\leq & \left\|C\left(\hat{\boldsymbol{l}}_{n_{k}}\right) \Pi_{n_{k}}-C(\underline{\boldsymbol{l}}) \Pi_{n_{k}}\right\|+\left\|C(\underline{\boldsymbol{l}}) \Pi_{n_{k}}-C(\underline{\boldsymbol{l}})\right\| \\
\leq & \left\|C\left(\hat{\boldsymbol{l}}_{n_{k}}\right)-C(\underline{\boldsymbol{l}})\right\|+\left\|C(\underline{\boldsymbol{l}}) \Pi_{n_{k}}-C(\underline{\boldsymbol{l}})\right\| .
\end{aligned}
$$

It follows that

$$
\lim _{k \rightarrow \infty}\left\|C_{n_{k}}\left(\hat{\boldsymbol{l}}_{n_{k}}\right) \Pi_{n_{k}}-C(\underline{\boldsymbol{l}})\right\|=0 .
$$

Therefore, $\left(A_{n_{k}}, C_{n_{k}}\left(\hat{l}_{n_{k}}\right), G_{n_{k}}\right), k=1,2, \ldots$, is a sequence of approximations for $(A, C(\underline{l}), G)$ that satisfies assumptions (H1)(H3). Theorem 4.2 then implies that

$$
\lim _{k \rightarrow \infty}\left\|P_{s s}^{\left(n_{k}\right)}\left(\hat{\boldsymbol{l}}_{n_{k}}\right) \Pi_{n_{k}}-P_{s s}(\underline{\boldsymbol{l}})\right\|_{1}=0,
$$

which further implies that

$$
\lim _{k \rightarrow \infty} \hat{\mu}_{n_{k}}=\left\|P_{s s}(\underline{\boldsymbol{l}})\right\|_{1} \geq \hat{\mu} .
$$

Thus, $\liminf _{n \rightarrow \infty} \hat{\mu}_{n} \geq \hat{\mu}$ and

$$
\lim _{n \rightarrow \infty} \hat{\mu}_{n}=\hat{\mu},
$$

which is (32).

This and (33) also imply that

$$
\hat{\mu}=\left\|P_{s s}(\underline{\boldsymbol{l}})\right\|_{1},
$$

that is, $\underline{\boldsymbol{l}}$ is an optimal sensor location. Since the sequence $\left\{\hat{\boldsymbol{l}}_{n_{k}}\right\}_{k=1}^{\infty}$ was an arbitrary convergent subsequence of $\left\{\hat{\boldsymbol{l}}_{n}\right\}_{n=1}^{\infty}$ it follows that any convergent subsequence converges to an optimal sensor location.

Thus, approximations can be used to obtain filters and determine optimal sensor locations to arbitrary accuracy. The theory developed in this and the previous section is applied to several examples in the next section.

\section{EXAMPLES}

\section{A. One-dimensional diffusion}

A one-dimensional diffusion equation with white Gaussian noise disturbance is considered:

$$
\begin{aligned}
& \frac{\partial z}{\partial t}=\sigma \frac{\partial^{2} z}{\partial x^{2}}+g(x) \eta(t), \quad 0 \leq x \leq 1, t \geq 0 \\
& \frac{\partial z}{\partial x}(0, t)=0, \quad \frac{\partial z}{\partial x}(1, t)=0 \\
& z(x, 0)=z_{0}(x)
\end{aligned}
$$

where $\sigma$ is the constant diffusivity, $g(x)$ models the shape of the spatially distributed disturbance, and $\eta(t)$ is assumed to be a real-valued white Gaussian noise, such that

$$
w(t)=\int_{0}^{t} \eta(s) d s
$$

is a Wiener process of incremental covariance $Q$. The physical interpretation of $z$ depends on the application and its value is with respect to a reference state. For example, in the case of thermal diffusion, $z$ is temperature, and temperature in Celsius is with reference to the freezing point of water.

Let the state space $\mathscr{Z}=L^{2}(0,1)$, and $A=\sigma \frac{\partial^{2}}{\partial x^{2}}$ with domain

$$
\mathscr{D}(A)=\left\{h \in \mathscr{H}^{2}(0,1): h^{\prime}(0)=h^{\prime}(1)=0\right\} \subset \mathscr{Z} .
$$

The state-space representation for the equation (34) is

$$
d z(t)=A z(t) d t+G d w(t), z(0)=z_{0}, t \geq 0,
$$

where $G \in \mathscr{L}(\mathbb{R}, \mathscr{Z})$ is defined by, for some $g \in \mathscr{Z}$,

$$
G \alpha=\alpha g(x), \text { for } \alpha \in \mathbb{R} .
$$

The operator $A$ generates an analytic $C_{0}$-semigroup $T(t)$, so that the solution to (35) can be expressed as (see [10, Theorem 5.35])

$$
z(t)=T(t) z_{0}+\int_{0}^{t} T(t-s) G d w(s), t \geq 0 .
$$

Assume that there are $m$ identical sensors. Each sensor measures the average state over an interval of length $\Delta>0$. Write

$$
\boldsymbol{l}=\left(l_{1}, l_{2}, \ldots, l_{m}\right),
$$

and define

$$
c_{l_{j}}(x):=\left\{\begin{array}{ll}
1 / \triangle, & \left|x-l_{j}\right| \leq \frac{\Delta}{2} \\
0, & \text { otherwise }
\end{array},\right.
$$

for $j=1,2, \ldots, m$. With $m$ sensors centred at $x=l_{1}, l_{2}, \ldots, l_{m}$,

$$
C(\boldsymbol{l}) z=\left(\left\langle c_{l_{1}}, z\right\rangle,\left\langle c_{l_{2}}, z\right\rangle, \ldots,\left\langle c_{l_{m}}, z\right\rangle\right)^{T} .
$$

Let $v_{j}(t)(j=1,2, \ldots, m)$ represent the noise in $j$-th sensor. The noises $v_{1}(t), v_{2}(t), \ldots, v_{m}(t)$ are mutually independent real-valued Wiener processes, with

$$
E\left\{\left(v_{j}(t)-v_{j}(s)\right)^{2}\right\}=(t-s) r_{0}, j=1,2, \ldots, m
$$

for $t \geq s \geq 0$, where $r_{0} \in \mathbb{R}_{+}$. If the sensors are of high quality, then $r_{0} \in \mathbb{R}_{+}$is small, while $r_{0}$ is larger for low-quality sensors. The covariance of the measurement noise $v(t)$ is

$$
R=\operatorname{diag}\left(r_{0}, r_{0} \ldots r_{0}\right) \in \mathbb{R}^{m \times m} .
$$

The operator $A=\sigma \frac{\partial^{2}}{\partial x^{2}}$ has eigenfunctions $\phi_{0}(x)=1$, $\phi_{j}(x)=\sqrt{2} \cos (j \pi x)$ for $j \geq 1$, with corresponding eigenvalues $\lambda_{j}:=-\sigma j^{2} \pi^{2}, j \geq 0$. The eigenfunctions form an orthonormal basis for the state space $\mathscr{Z}=L_{2}(0,1)$. A Galerkin approximation obtained with first $n$ eigenfunctions of $A$ will be used in the calculations.

When using approximations of order $n \geq 4$, simulation results show that the optimal observability of the approximate systems over the set of posible sensor locations is 0 , reflecting the fact that the original model is at best only approximately 
observability. For more detail on this point in the context of controllability, see [28]. Observability is not a useful cost function.

The subsequent approximating systems satisfy the assumptions of Theorem 4.2 (see for example [20]) and so

$$
\lim _{n \rightarrow \infty}\left\|P_{s s}^{(n)} \Pi_{n}-P_{s s}\right\|_{1}=0 .
$$

For the simulations, the diffusivity $\sigma=0.1$, the approximation order $N=20$ and the coefficient of process noise covariance was $Q=10$. The sensors each had width of $\triangle=0.04$ and the sensors had 25 possible locations evenly distributed in $[0,1]$. The ARE's were solved using Matlab function 'care'. All the sensors were optimally placed using a dual version of the algorithm in [13] to calculate the sensor locations that minimized the steady-state estimation error covariance $\left\|P_{s s}\right\|_{1}$. The initial condition was

$$
z_{0}=a\left(2 x+x^{2}\right)
$$

where $a$ has normal distribution $\mathbf{N}(0,10)$ and was chosen using the Matlab function 'randn'. The stochastic differential equations were solved using the explicit Euler method [19]. time step size $\triangle t=0.001$, and the noise term $w_{\triangle t}(k)$, that has normal distribution $\boldsymbol{N}(0, Q \triangle t)$, was generated by the Matlab function 'randn' at each time step.

Three different disturbances were considered: spatially uniform, localized and a mixture of the two types.

Consider first the case where the noise on the state is evenly distributed in space:

$$
g(x)=1 .
$$

The initial condition (36) was scaled by $a=8.5886$. When using a single sensor, the value of $\left\|P_{S S}\right\|_{1}$ was computed for $r_{0}$ ranging from 0.1 to 2 . As shown in Figure $1(\mathrm{a}),\left\|P_{s s}\right\|_{1}$ is an increasing function of $r_{0}$ and it coincides well with

$$
f_{1}\left(r_{0}\right):=\tilde{C} \sqrt{r_{0}},
$$

in which the constant $\tilde{C} \approx 1.00$. Figure $1(\mathrm{~b})$ shows that $\left\|P_{s S}\right\|_{1}$ decreases as the sensor number $m$ increases. Calculations of $\left\|P_{s s}\right\|_{1}$ were done with $m$ optimally placed sensors, each which variance $r_{0}=2$, for various values of $m$. Let

$$
g_{1}(m):=C_{2} / \sqrt{m} \quad\left(C_{2} \approx 1.41\right)
$$

be the function defined continuously on the interval $[0,20]$. As shown in Figure 1(b), the curve of function $g_{1}$ fits with the value of $\left\|P_{s s}\right\|_{1}$ for $m=1,2, \ldots, 20$. For this example $\left\|P_{s s}\right\|_{1}$ is approximately proportional to the value of $\sqrt{r_{0} / m}$ :

$$
\left\|P_{s s}\right\|_{1} \approx C \sqrt{r_{0} / m}
$$

with $C=\tilde{C} \approx C_{2} / \sqrt{2}$. Figure 1(b) indicates that increasing the number of sensors can compensate for the inaccuracy of a sensor with a large noise variance. The estimates obtained by using a single sensor with noise variance $r_{0}=0.2$, and by using 15 sensors each with noise variance $r_{0}=2$, are compared in Figure 2 with the actual system state $z(t)$ at the middle point $x=0.5$ for $t \in[0,20]$. For a single sensor, the value of $\left\|P_{s s}\right\|_{1}$ was computed for different sensor locations, which is constant with respect to sensor location. Hence, there does not exist a unique optimal sensor location in this case. For 15 sensors, searching by the optimization algorithm from [13], the optimal sensor locations were found to be centered at

$\hat{\imath}=(0.02,0.10,0.14,0.22,0.30,0.38,0.42,0.50,0.58,0.62,0.70$, $0.78,0.86,0.90,0.98)$.

Using multiple sensors each with larger noise variance led to a better estimate than a single accurate sensor.

Now consider a localized disturbance illustrated in Figure 3:

$$
g(x)=\operatorname{sech}(100(x-0.2)) .
$$

The initial condition (36) was scaled by $a=3.3261$. By computing the value of $\left\|P_{S S}\right\|_{1}$ for the case that using a single sensor $(m=1)$ with variance $r_{0} \in[0.1,2]$, an ascending curve is derived, as shown in Figure 4(a). It again coincides well with a square root function:

$$
f_{2}\left(r_{0}\right):=C_{3} \sqrt{r_{0}} \quad\left(C_{3} \approx 0.09\right) .
$$

The value of $\left\|P_{s s}\right\|_{1}$ as a function of the number of sensors fits well with the curve of a decreasing function

$$
g_{2}(m):=C_{4} / \sqrt{m} \quad\left(C_{4} \approx 0.14\right),
$$

as shown in Figure 4(b). Hence the same proportional relation (37) but with constant $C=C_{3} \approx C_{4} / \sqrt{2}$ appears to hold. Comparisons of the actual system state with estimates made by a single sensor with variance $r_{0}=0.2$ and by 15 sensors with variance $r_{0}=2$ are displayed in Figure 5. For a single sensor, the value of $\left\|P_{s s}\right\|_{1}$ was computed for different sensor locations; the optimal sensor location is centered at $\hat{l}=0.18$, around where the localized disturbance is. For 15 sensors, searching by the optimization algorithm from [13], the optimal sensor locations were found to be centered at

$\hat{\imath}=(0.02,0.06,0.10,0.14,0.18,0.22,0.26,0.30,0.34,0.38,0.42$,

$$
0.46,0.50,0.54,0.58) \text {. }
$$

The errors for the two estimates are similar.

A third disturbance that includes both the evenly distributed disturbance and the spatially localized disturbance:

$$
g(x)=0.5 \operatorname{sech}(100(x-0.2))+0.5,
$$

(Figure 6) was also considered. The initial condition (36) was scaled by $a=6.8543$. The same computations as for the previous two disturbances were done. The value of $\left\|P_{S S}\right\|_{1}$ as the sensor noise covariance is changed are shown in Figure 7. Two curves

$$
\begin{gathered}
f_{3}\left(r_{0}\right):=C_{5} \sqrt{r_{0}} \quad\left(C_{5} \approx 1.57\right), \\
g_{3}(m):=C_{6} / \sqrt{m} \quad\left(C_{6} \approx 2.25\right) .
\end{gathered}
$$

were fit to the plots in Figure 7(a) and 7(b) respectively. The same square root relation as for other disturbances seems to hold with constant $C=C_{5} \approx C_{6} / \sqrt{2}$. Estimation using one sensor with noise variance $r_{0}=0.2$ is compared with that obtained using 15 sensors each with noise variance $r_{0}=2$ in Figure 8. For a single sensor, the value of $\left\|P_{S S}\right\|_{1}$ was computed for different sensor locations; the optimal sensor location is centered at $\hat{l}=0.18$, around where the disturbance peaks. For 
15 sensors, searching by the optimization algorithm from [13], the optimal sensor locations were found to be centered at

$$
\begin{aligned}
\hat{\boldsymbol{l}}= & (0.02,0.06,0.10,0.14,0.18,0.22,0.26,0.30,0.34,0.38,0.42, \\
& 0.46,0.50,0.54,0.58)
\end{aligned}
$$

The accuracy of the estimate obtained with multiple poor sensors is better than that obtained with one accurate sensor.

\section{B. Simply supported Euler-Bernoulli beam}

Consider an Euler-Bernoulli beam of length 1, with KelvinVoigt damping. Let $f(t, x)$ denote the deflection of the beam at time $t$ and position $x$. The beam deflection is described by the partial differential equation

$$
\frac{\partial^{2} f}{\partial t^{2}}+\frac{\partial^{4} f}{\partial x^{4}}+c_{d} \frac{\partial^{5} f}{\partial x^{4} \partial t}=g(x) \eta(t), \quad t \geq 0,0<x<1,
$$

where $c_{d}$ is the damping parameter, $g(x)$ models the shape of the spatially distributed disturbance, and $\eta(t)$ is a real-valued white Gaussian noise, such that

$$
w(t)=\int_{0}^{t} \eta(s) d s
$$

is a Wiener process of incremental covariance $Q$. Assume the initial condition

$$
f(0, x)=f_{0}(x), \frac{\partial}{\partial t} f(0, x)=f_{1}(x),
$$

and simply supported boundary conditions

$$
\begin{aligned}
& f(t, 0)=0, \quad \frac{\partial^{2} f(t, 0)}{\partial x^{2}}+c_{d} \frac{\partial^{3} f(t, 0)}{\partial x^{2} \partial t}=0, \\
& f(t, 1)=0, \quad \frac{\partial^{2} f(t, 1)}{\partial x^{2}}+c_{d} \frac{\partial^{3} f(t, 1)}{\partial x^{2} \partial t}=0 .
\end{aligned}
$$

Let

$$
\mathscr{H}_{s}(0,1)=\left\{f \in \mathscr{H}^{2}(0,1): f(0)=f(1)=0\right\}
$$

and the state space $\mathscr{Z}=\mathscr{H}_{s}(0,1) \times L^{2}(0,1)$, with state $\mathbf{z}:=$ $\left(f, f_{t}\right)^{T} \in \mathscr{Z}$. Equation (38) can be written in the state-space form

$$
d \mathbf{z}(t)=A \mathbf{z}(t) d t+G d w(t), \mathbf{z}(0)=\mathbf{z}_{0}, t \geq 0,
$$

where

$A=\left(\begin{array}{cc}0 & 1 \\ -\frac{\partial^{4}}{\partial x^{4}} & -c_{d} \frac{\partial^{4}}{\partial x^{4}}\end{array}\right), G=\left(\begin{array}{c}0 \\ g(x)\end{array}\right), \mathbf{z}_{0}=\left(\begin{array}{c}f_{0}(x) \\ f_{1}(x)\end{array}\right)$,

with domain

$$
\mathscr{D}(A)=\left\{\mathbf{z}=\left(z_{1}, z_{2}\right) \in \mathscr{Z}: z_{1}^{\prime \prime} \in H_{s}(0,1), z_{2}^{\prime \prime} \in H_{s}(0,1)\right\} .
$$

Each sensor measures average deflection over a small interval of length $\triangle>0$. Write

$$
\boldsymbol{l}=\left(l_{1}, l_{2}, \ldots, l_{m}\right)
$$

and define

$$
c_{l_{j}}(x):= \begin{cases}1 / \triangle, & \left|x-l_{j}\right| \leq \frac{\Delta}{2} \\ 0, & \text { otherwise }\end{cases}
$$

for $j=1,2, \ldots, m$. With $m$ sensors centred at $x=l_{1}, l_{2}, \ldots, l_{m}$,

$$
\begin{aligned}
C(l) \boldsymbol{z}(t)= & \left(\int_{0}^{1} c_{l_{1}}(x) f(t, x) d x, \int_{0}^{1} c_{l_{2}}(x) f(t, x) d x, \ldots,\right. \\
& \left.\int_{0}^{1} c_{l_{n}}(x) f(t, x) d x\right)^{T},
\end{aligned}
$$

and the output is

$$
y(t)=\int_{0}^{t} C(\boldsymbol{l}) \boldsymbol{z}(s) d s+v(t),
$$

where $v(t)$ is an $\mathbb{R}^{m}$-valued Wiener process of incremental covariance

$$
R=\operatorname{diag}\left(r_{0}, r_{0} \ldots r_{0}\right) \in \mathbb{R}^{m \times m} .
$$

The eigenfunctions of $A$ are used as the basis for a Galerkin approximation. This approximation satisfies the assumptions of Theorem 4.2; see for example [20] for details. As for the diffusion problem, if more than 8 modes are used in the approxmation $(n \geq 16)$, optimal observability of the approximations over the set of posible sensor locations is 0 , reflecting the fact that the original model is at best only approximately observability. See [28] for details.

For simulations, the parameter values are $c_{d}=0.0001, \triangle=$ $0.02,30$ modes were used, and the initial condition

$$
f_{0}(x)=0.25-(x-0.5)^{2}, f_{1}(x)=0,0<x<1 .
$$

The stochastic differential equations were solved using the implicit Euler method:

$$
z_{n}\left(t_{k+1}\right)=z_{n}\left(t_{k}\right)+A_{n} z_{n}\left(t_{k+1}\right) \triangle t+G_{n} w_{\triangle t}(k),
$$

where the time step size $\triangle t=0.001$, and the noise term $w_{\triangle t}(k)$, that has normal distribution $\boldsymbol{N}(0, Q \triangle t)$, was generated by the Matlab function 'randn' at each time step. It follows from (40) that

$$
\left(I_{n}-A_{n} \triangle t\right) z_{n}\left(t_{k+1}\right)=z_{n}\left(t_{k}\right)+G_{n} w_{\triangle t}(k) .
$$

The equation was then solved using Matlab function 'linsolve'. Again, three different spatial distributions of the noise were considered. For all cases, the process noise $\tilde{Q}=1$.

Consider first

$$
g(x)=1 .
$$

Estimates obtained using a single high-quality sensor are compared with estimates obtained using multiple relatively low-quality sensors. The estimates obtained by using a single sensor with $r_{0}=0.002$ and by using 15 sensors each with $r_{0}=0.02$ are compared in Figure 10 with the actual system state $z(t)$ at the middle point $x=0.5$ for $t \in[0,15]$. The optimal location for the single sensor is $\hat{l}=0.51$; the optimal locations for 15 sensors are

$$
\begin{aligned}
\hat{l}= & (0.35,0.37,0.39,0.41,0.43,0.45,0.47,0.49,0.51,0.53,0.55, \\
& 0.57,0.59,0.61,0.63) .
\end{aligned}
$$

For a localized disturbance

$$
g(x)=\operatorname{sech}(100(x-0.2)),
$$

comparisons of the actual system state with estimates made by a single sensor with $r_{0}=0.002$ and by 15 sensors with 
$r_{0}=0.02$ are displayed in Figure 11. The estimation errors are similar. The optimal location for the single sensor is centred at $\hat{l}=0.49$; the optimal locations for 15 sensors are

$\hat{\imath}=(0.41,0.43,0.45,0.47,0.49,0.51,0.53,0.55,0.57,0.59,0.61$, $0.63,0.65,0.67,0.69)$.

Then, consider a mixed disturbance that includes both the evenly distributed disturbance and the spatially localized disturbance:

$$
g(x)=0.5 \operatorname{sech}(100(x-0.2))+0.5,
$$

The estimate obtained using one sensor with $r_{0}=0.002$ is compared with that obtained using 15 sensors each with $r_{0}=$ 0.02 in Figure 12. The optimal location for the single sensor is centred at $\hat{l}=0.49$; the optimal locations for the 15 sensor are

$\hat{\boldsymbol{l}}=(0.37,0.39,0.41,0.43,0.45,0.47,0.49,0.51,0.53,0.55,0.57$, $0.59,0.61,0.63,0.65)$.

All simulation results illustrated in Figure 10-12 indicate that increasing the number of sensors can compensate for higher sensor noise.

\section{Two-dimensional diffusion}

Let $\Omega$ be the L-shaped region in $\mathbb{R}^{2}$ shown in Figure 13 . Consider two-dimensional diffusion on $\Omega$ with white Gaussian noise disturbance:

$$
\begin{aligned}
& z_{t}=\sigma\left(\frac{\partial^{2} z}{\partial x_{1}^{2}}+\frac{\partial^{2} z}{\partial x_{2}^{2}}\right)+g\left(x_{1}, x_{2}\right) \eta(t),\left(x_{1}, x_{2}\right) \in \Omega, \\
& \left.z\left(t, x_{1}, x_{2}\right)\right|_{\partial \Omega}=0, \\
& z\left(0, x_{1}, x_{2}\right)=z_{0}\left(x_{1}, x_{2}\right),
\end{aligned}
$$

where $\sigma$ is the constant diffusivity, $g\left(x_{1}, x_{2}\right)$ models the shape of the spatially distributed disturbance, and $\eta(t)$ is assumed to be a real-valued white Gaussian noise, such that

$$
w(t)=\int_{0}^{t} \eta(s) d s
$$

is a Wiener process of incremental covariance $Q$. The state space is $\mathscr{Z}=L^{2}(\Omega)$, and the sensor noise operator $G \in$ $\mathscr{L}(\mathbb{R}, \mathscr{Z})$ is of the form that for $k \in \mathbb{R}$,

$$
G k=k g\left(x_{1}, x_{2}\right) .
$$

The infinitesimal generator $A=\sigma \nabla^{2}$ is defined in the weak form (see $[14, \S 1.5 .3])$

$$
A z=\sigma \phi_{z},
$$

where $\phi_{z} \in \mathscr{H}^{-1}(\Omega)$ satisfies

$$
\int_{\Omega} \phi_{z} \varphi d x_{1} d x_{2}=-\int_{\Omega} \nabla z \cdot \nabla \varphi d x_{1} d x_{2}
$$

for all $\varphi \in \mathscr{H}_{0}^{1}(\Omega)$, with the domain

$$
\mathscr{D}(A)=\left\{z \in \mathscr{H}_{0}^{1}(\Omega): \nabla^{2} z \in L^{2}(\Omega)\right\} \subset \mathscr{Z} .
$$

Each sensor measures the average of $z(t)$ over a small square of side length $\Delta>0$. Write

$$
\boldsymbol{l}=\left(l_{1}, l_{2}, \ldots, l_{m}\right) \in \mathbb{R}^{2 \times m},
$$

and define

$c_{l_{j}}\left(x_{1}, x_{2}\right):= \begin{cases}1 / \triangle^{2}, & \text { if }\left|x_{1}-l_{j}(1)\right| \leq \frac{\Delta}{2},\left|x_{2}-l_{j}(2)\right| \leq \frac{\Delta}{2} \\ 0, & \text { otherwise, }\end{cases}$ for $j=1,2, \ldots, m$. With $m$ sensors centred at $x=l_{1}, l_{2}, \ldots, l_{m}$,

$$
C(l) z=\left(\left\langle c_{l_{1}}, z\right\rangle,\left\langle c_{l_{2}}, z\right\rangle, \ldots,\left\langle c_{l_{m}}, z\right\rangle\right)^{T},
$$

the measurement is

$$
y(t)=\int_{0}^{t} C(\boldsymbol{l}) z(s) d s+v(t),
$$

where $v(t)$ is an $\mathbb{R}^{m}$-valued Wiener process of incremental covariance

$$
R=\operatorname{diag}\left(r_{0}, r_{0} \ldots r_{0}\right) \in \mathbb{R}^{m \times m} .
$$

A standard finite element method with linear basis functions [2] was used to approximate the two-dimensional diffusion equation. A triangular mesh of the L-shaped region $\Omega$ was created and refined using Matlab functions 'initmesh' and 'refinemesh'. The mesh is shown in Figure 13. Suppose there are $n$ interior nodes $\left\{v_{j}: j=1,2, \ldots, n\right\}$ in the mesh. Basis functions $\left\{\phi_{j}: j=1,2, \ldots, n\right\} \subset \mathscr{V}$ are chosen to be linear spline functions such that

$$
\phi_{j}\left(v_{k}\right)=\delta_{j k}, j, k=1,2, \ldots, n,
$$

where $\delta_{j k}$ is the Kronecker delta.

All the assumptions of Theorem 4.2 are again satisfied; see [16] for a similar example.

For simulations, the triangular mesh shown in Figure 13 was used, which contains $N=526$ nodes. The mesh size is 0.1 , that is, the maximal length of any triangle side. The parameters are $\sigma=0.1, Q=0.01, \triangle=0.1$, and the initial condition

$$
z_{0}\left(x_{1}, x_{2}\right)=\operatorname{sech}\left(\left(10\left(x_{1}-1.5\right)^{2}+50\left(x_{2}-0.3\right)^{2}\right)\right) .
$$

The implicit Euler method was used to solve the resulting stochastic differential equation, with time step size $\triangle t=$ 0.001 , and the noise term $w_{\triangle t}(k)$, that has normal distribution $\boldsymbol{N}(0, Q \triangle t)$, was generated by the Matlab function 'randn' at each time step.

Consider a spatially localized disturbance centered at $x=$ $(1.5,1.5)$ (see Figure 14):

$$
g(x)=\operatorname{sech}\left(100\left(\left(x_{1}-1.5\right)^{2}+\left(x_{2}-1.5\right)^{2}\right)\right) .
$$

Using a single sensor, the optimal sensor location is centered at $x=(1.55,1.55)$, near the maximum of the disturbance. When using 25 sensors, the optimal sensor locations are still gathered at around the location of the disturbance (see Figure 15). The estimates obtained by using a single sensor with $r_{0}=0.0002$, and by using 25 sensors each with $r_{0}=0.002$, are compared in Figure 16. Again, the two estimates are similar, which indicates that using more sensors can compensate for large noise in sensors. 


\section{CONCLUSIONS}

It was shown that the nuclear norm of the solution to the operator Riccati equation is the steady-state minimum error variance of an estimate. Earlier results and an algorithm on linear-quadratic optimal actuator location extend in a straightforward way to locate sensors that minimize the error variance.

Using the value of $\left\|P_{s S}\right\|_{1}$ as the optimality criterion, the effects of sensor noise on state estimation were investigated. It was proven formally that reducing the sensor noise improves the estimate, and so does increasing the number of sensors. Thus, a larger number of sensors can compensate for poor sensor quality.

Three examples were examined: one-dimensional diffusion, simply supported Euler-Bernoulli beam with KelvinVoigt damping, and two-dimensional diffusion. For the onedimensional diffusion equation, three different disturbances were considered: spatially evenly-distributed disturbance, spatially localized disturbance and mixed disturbance that combines the evenly distributed disturbance with a spatially localized disturbance. Assuming that all the selected sensors are optimally placed, accuracy of the estimation does depends on sensor quality, as expected. Furthermore, using a larger number of poor quality sensors, that is those with large noise variance, leads to an estimator with accuracy comparable to that with a single good quality sensor. Similar results are also observed for the simply supported Euler-Bernoulli beam and the two-dimensional diffusion equation. Also, for the one-dimensional diffusion equation, there is an approximately square root relation between $\left\|P_{s s}\right\|_{1}$ and the noise variance, as well as between $\left\|P_{S S}\right\|_{1}$ and the number of sensors. A theoretical justification for this observation has not been established.

Current research effort is concentrating on extending this work on sensor choice and location to nonlinear systems.

\section{REFERENCES}

[1] Antonios Armaou and Michael A. Demetriou. Optimal actuator/sensor placement for linear parabolic PDEs using spatial $\mathscr{H}_{2}$ norm. Chemical Engineering Science, 61(22):7351 - 7367, 2006.

[2] Owe Axelsson and Vincent Allan Barker. Finite Element Solution of Boundary Value Problems: Theory and Computation. Academic Press, Inc., Orlando, FL, 1984.

[3] Alain Bensoussan. Optimization of sensors' location in a distributed filtering problem, volume 294 of Lecture Notes in Mathematics, pages 62-84. Springer Berlin Heidelberg, Berlin, Heidelberg, 1972.

[4] J. A. Burns and C. N. Rautenberg. The infinitedimensional optimal filtering problem with mobile and stationary sensor networks. Numerical Functional Analysis and Optimization, 36(2):181-224, 2015.

[5] John A. Burns and Carlos N. Rautenberg. Solutions and approximations to the riccati integral equation with values in a space of compact operators. SIAM Journal on Control and Optimization, 53(5):2846-2877, 2015.

[6] G. Colantuoni and L.Padmanabhan. Optimal sensor selection in sequential estimation problems. International Journal of Control, 28(6):821-845, 1978. (a)

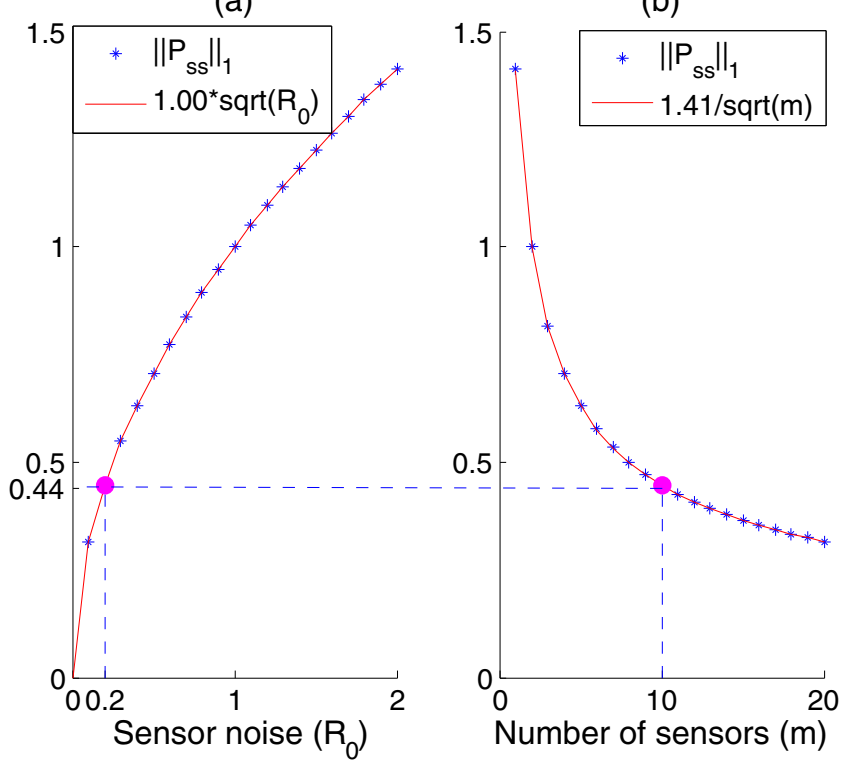

Fig. 1. Uniform disturbance $g(x)=1$. (a) $\left\|P_{s s}\right\|_{1}$ is an increasing function of $R=r_{0}$, coincides well with the curve $f_{1}\left(r_{0}\right)=\tilde{C} \sqrt{r_{0}}, \tilde{C} \approx 1.00$; (b) $\left\|P_{s s}\right\|_{1}$ is an decreasing function of the number of sensors $(m)$ with $r_{0}=2$. The curve is matched by $g_{1}(m)=C_{2} / \sqrt{m}, C_{2} \approx 1.41$

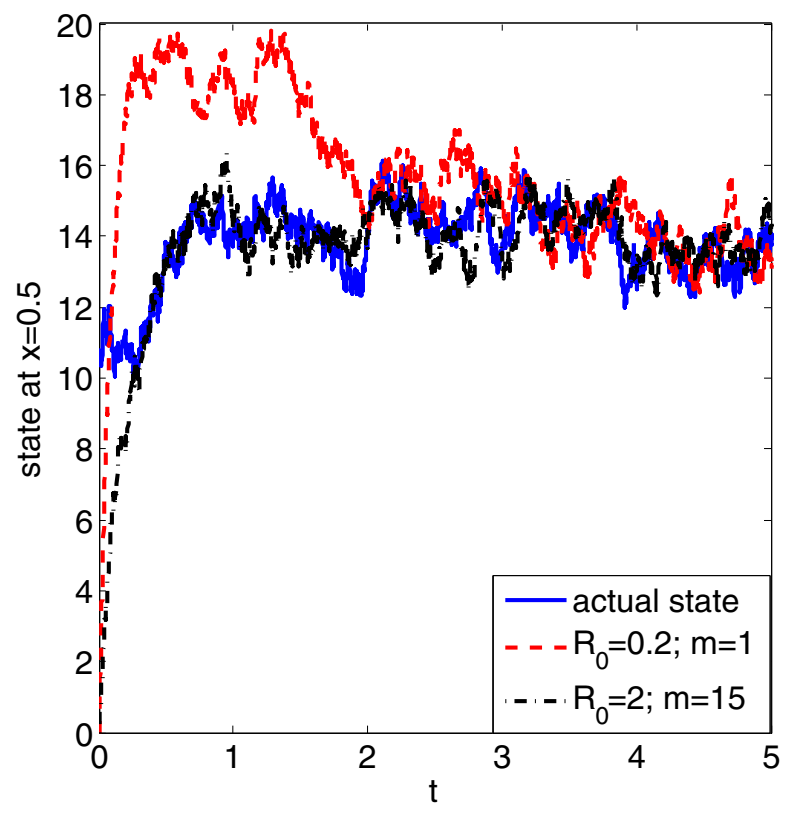

Fig. 2. Comparison of the actual state at $x=0.5, t \in[0,5]$ for $g(x)=1$ with estimates using a single sensor with noise variance $r_{0}=0.2$ and 15 sensors each with $r_{0}=2$. 


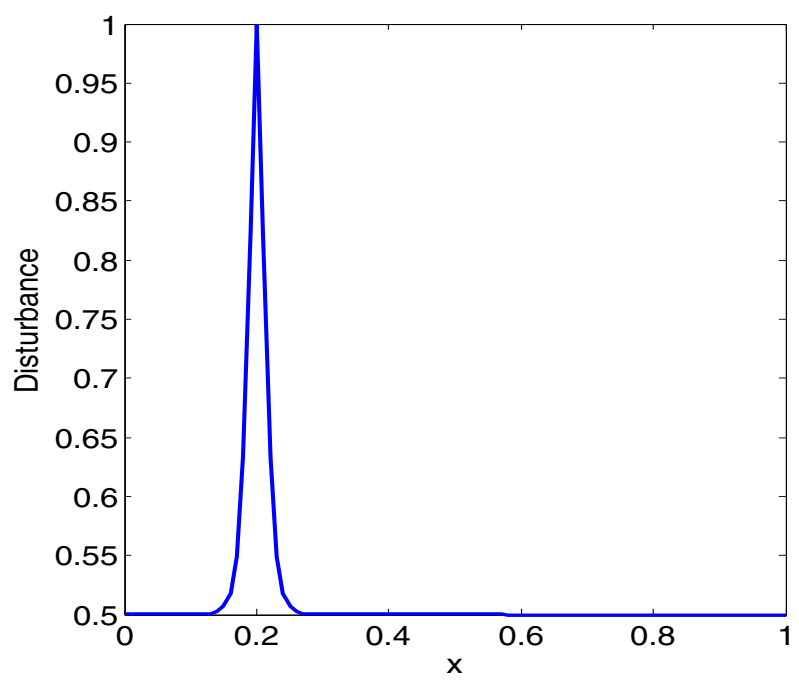

Fig. 3. Disturbance spatially localized at around $x=0.2$. (a)

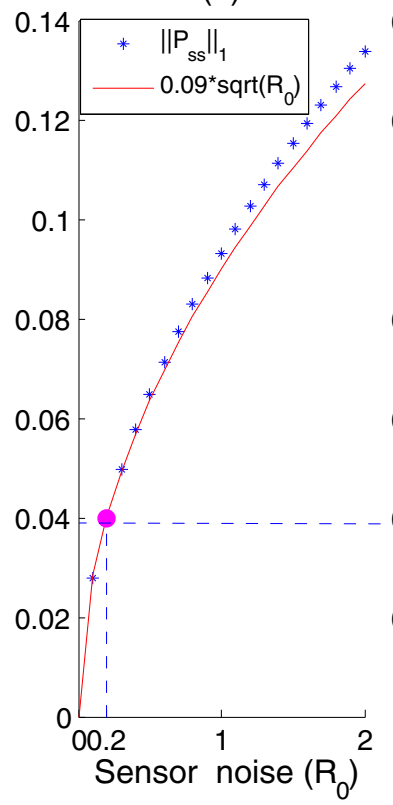

(b)

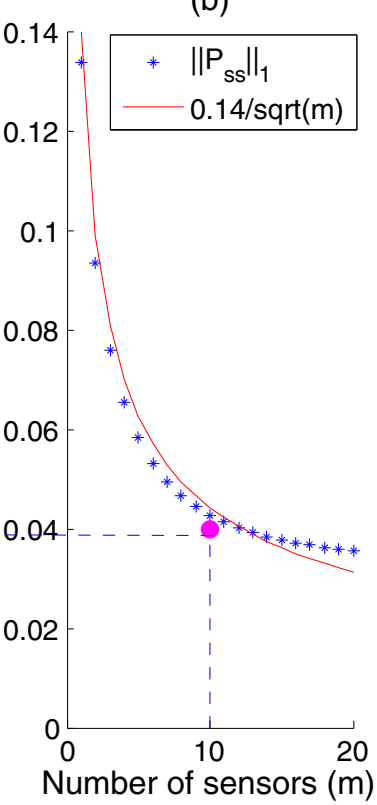

Fig. 4. Spatially localized disturbance. (a) $\left\|P_{S S}\right\|_{1}$ is an increasing function of $R=r_{0}$. It coincides $f_{2}\left(r_{0}\right)=C_{3} \sqrt{r_{0}}, C_{3} \approx 0.09$; (b) $\left\|P_{s s}\right\|_{1}$ is an decreasing function of the number of sensors $(m)$ with fixed $r_{0}=2$. It is matched by the curve $g_{2}(m)=C_{4} / \sqrt{m}, C_{4} \approx 0.14$.

[7] Ruth F. Curtain. Infinite-dimensional filtering. SIAM J. Control, 13:89-104, 1975.

[8] Ruth F. Curtain. A survey of infinite-dimensional filtering. SIAM Review, 17(3):395-411, 1975.

[9] Ruth F. Curtain, Kalle Mikkola, and Amol Sasane. The Hilbert-Schmidt property of feedback operators. J. Math. Anal. Appl., 329(2):1145-1160, 2007.

[10] Ruth F. Curtain and Anthony J. Pritchard. Infinite dimensional linear systems theory, volume 8 of Lecture Notes in Control and Information Sciences. Springer-

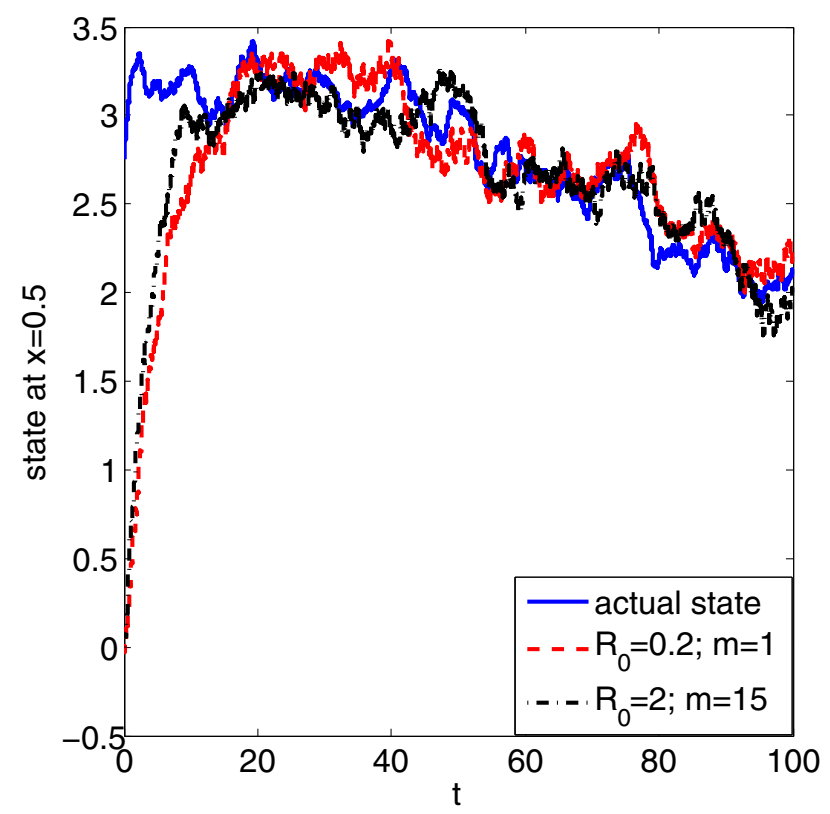

Fig. 5. Comparison of the actual and estimated state at $x=0.5$ with $g(x)$ a spatially localized disturbance. One estimate is obtained using a single sensor with noise variance $r_{0}=0.2$, while the other uses 15 sensors each with $r_{0}=2$.

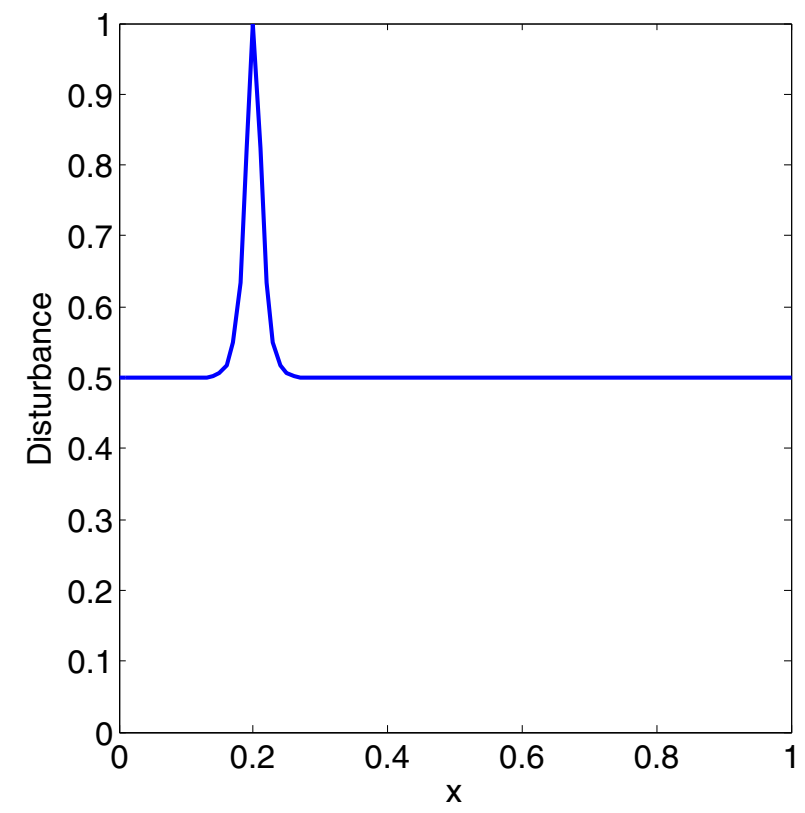

Fig. 6. Mixed disturbance $d(x)=0.5 \operatorname{sech}(100(x-0.2))+0.5$. 
(a)

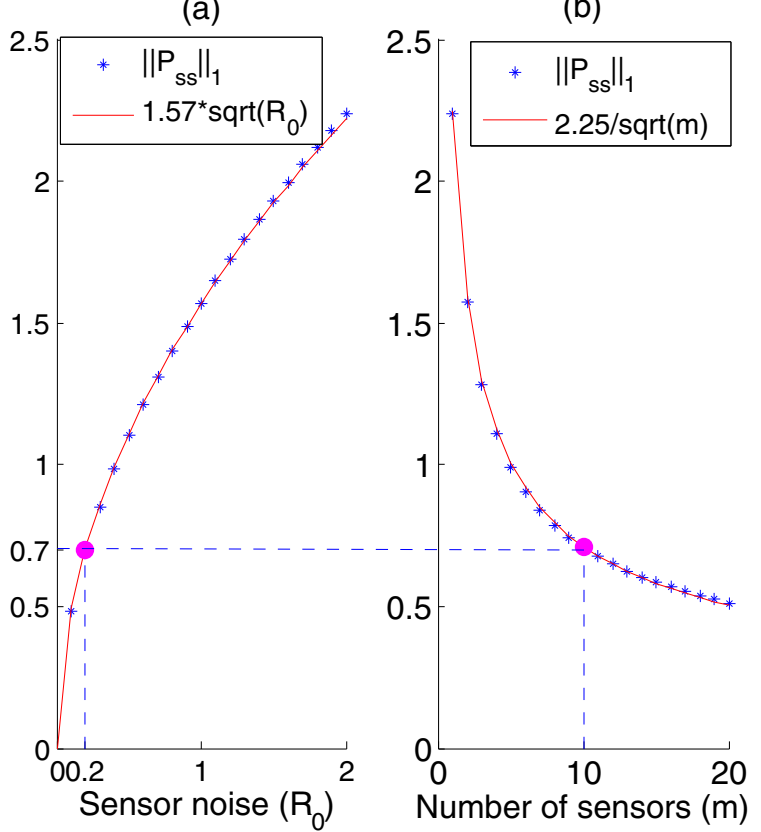

Fig. 7. Mixed disturbance. (a) $\left\|P_{s s}\right\|_{1}$ is an increasing function of $R=r_{0}$ and the behaviour matches the curve $f_{3}\left(r_{0}\right)=C_{5} \sqrt{r_{0}}, C_{5} \approx 1.57$; (b) $\left\|P_{s s}\right\|_{1}$ is an decreasing function of the number of sensors $(m)$ and matches the curve $g_{3}(m)=C_{6} / \sqrt{m}, C_{6} \approx 2.25$

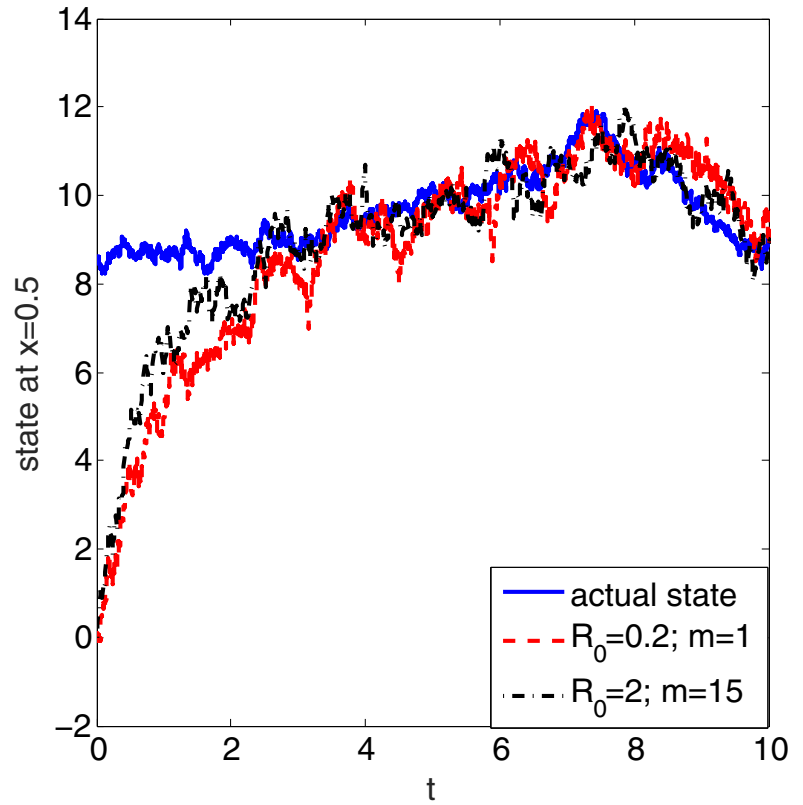

Fig. 8. Mixed disturbance. Comparison of the actual and estimated state at $x=0.5$. The two estimates are obtained from a single sensor with noise variance $r_{0}=0.2$ and 15 sensors each with $r_{0}=2$.
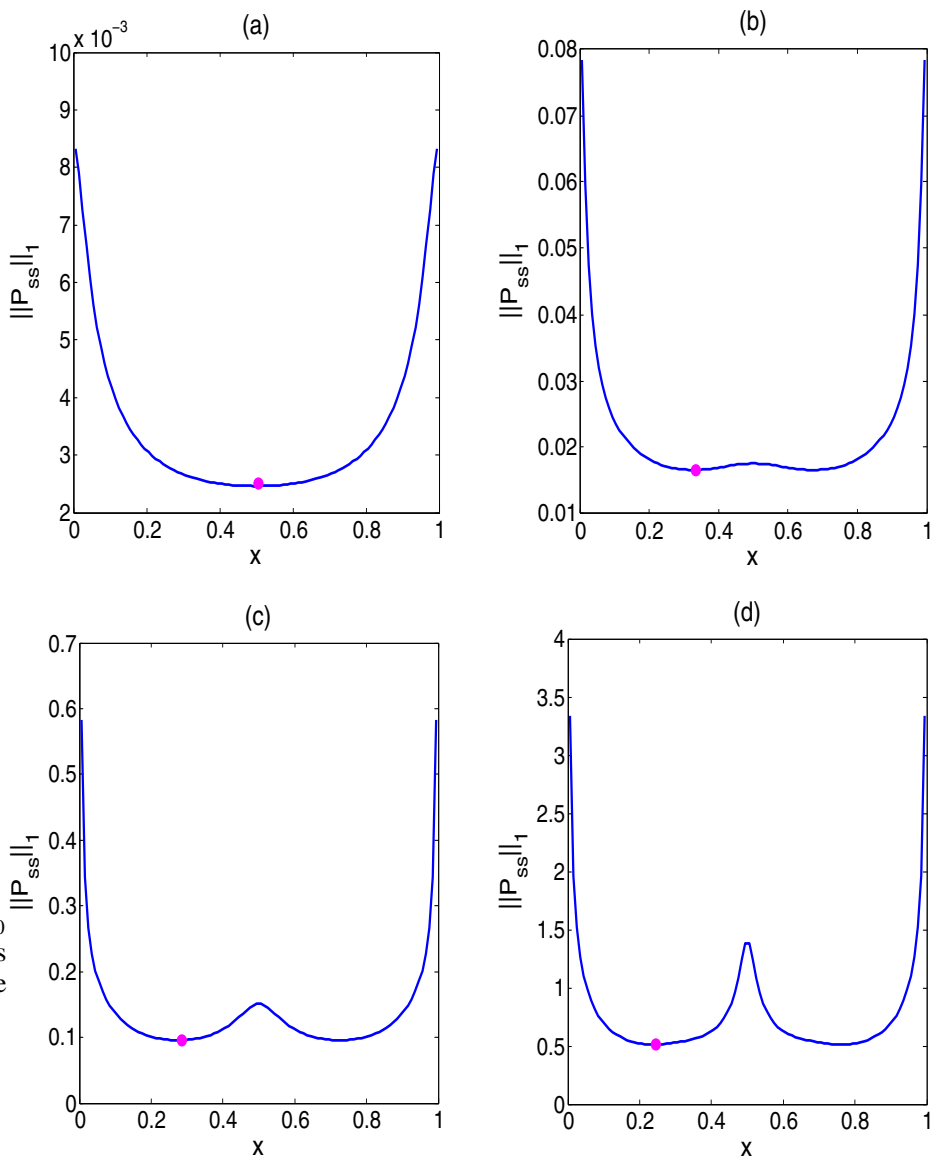

Fig. 9. The value of $\left\|P_{s s}\right\|_{1}$ for different sensor location, with noise parameters $r_{0}=0.0001$, and (a) $Q=0.1$, (b) $Q=1$, (c) $Q=10$, (d) $Q=100$; the magenta dots indicate the optimal sensor locations.

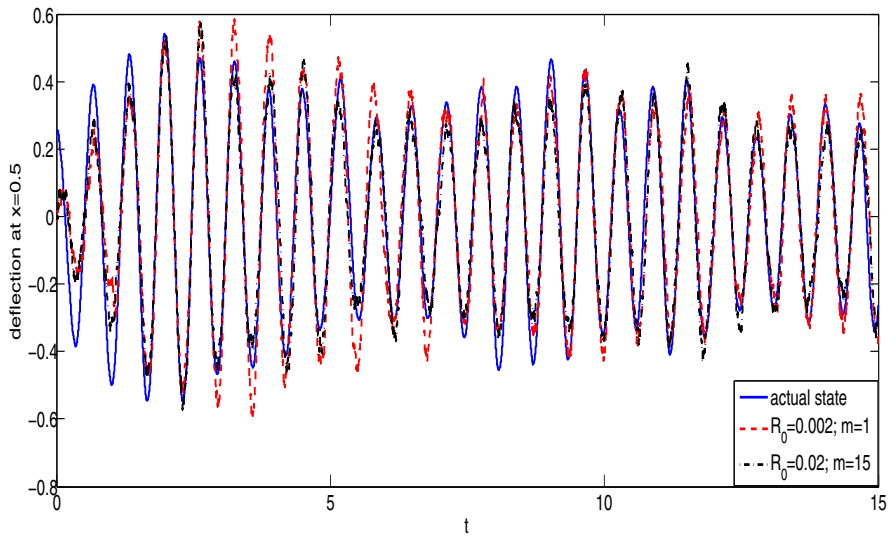

Fig. 10. Uniform disturbance $g(x)=1$. Comparison of the actual state at $x=0.5, t \in[0,15]$ with estimates using a single sensor with noise variance $r_{0}=0.002$ and 15 sensors each with $r_{0}=0.02$. 


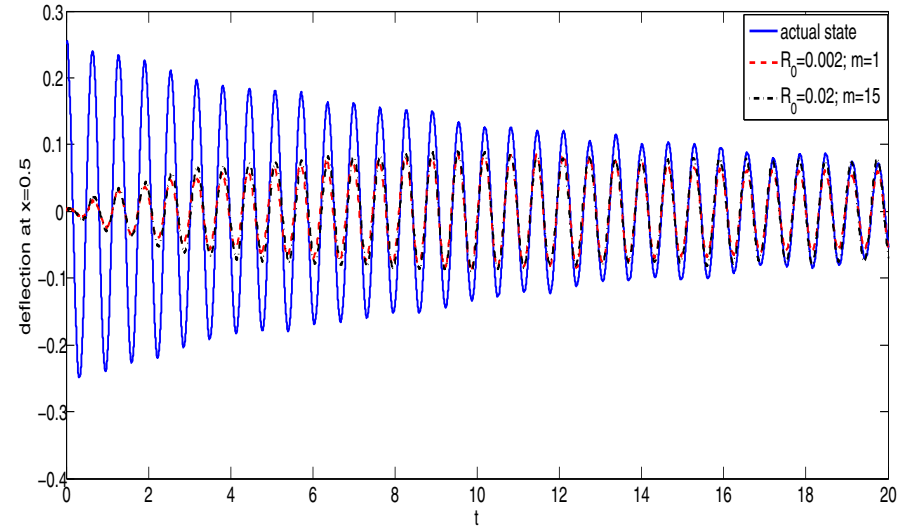

Fig. 11. Spatially localized disturbance. Comparison of the actual state at $x=0.5, t \in[0,10]$ with estimates using a single sensor with noise variance $r_{0}=0.02$ and 15 sensors each with $r_{0}=0.2$.

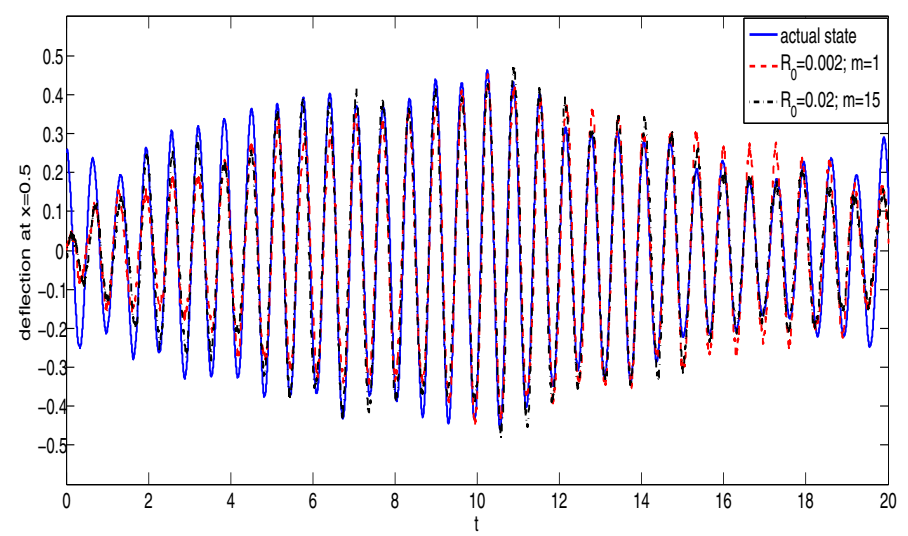

Fig. 12. Mixed disturbance. Comparison of the actual state at $x=0.5$, $t \in[0,20]$ with estimates using a single sensor with noise variance $r_{0}=0.002$ and 15 sensors each with $r_{0}=0.02$.

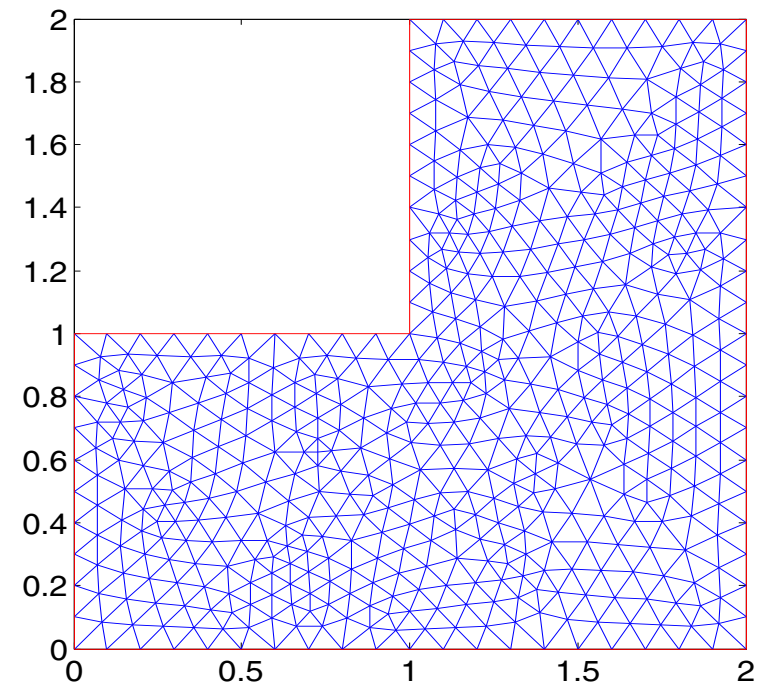

Fig. 13. Triangular mesh of $\Omega$

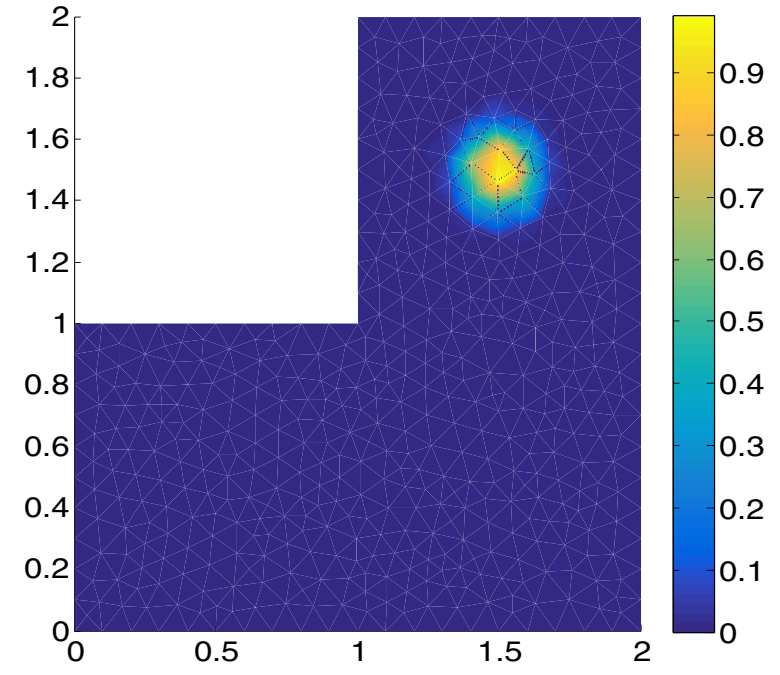

Fig. 14. Disturbance spatially localized around $x=(1.5,1.5)$

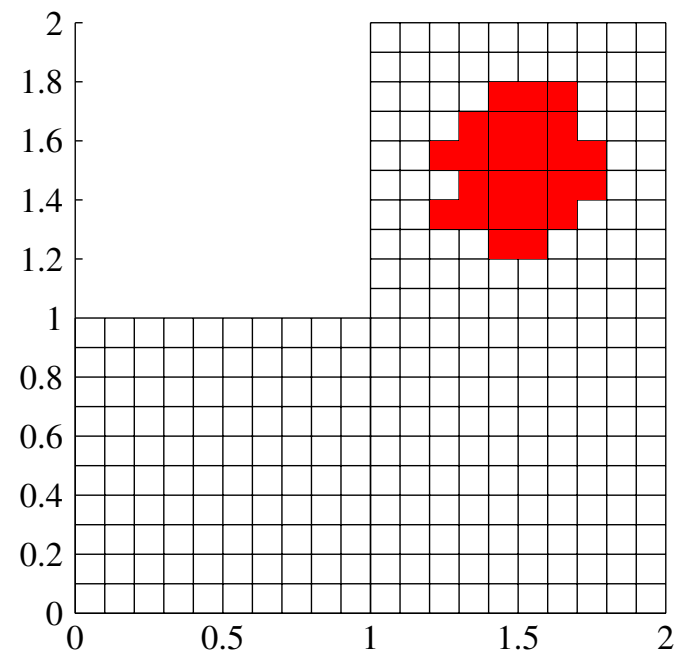

Fig. 15. Optimal location for 25 sensors

Verlag, Berlin-New York, 1978.

[11] Ruth F. Curtain and Leiba Rodman. Comparison theorems for infinite-dimensional Riccati equations. Systems \& Control Letters, 15(2):153-159, 1990.

[12] Ruth F. Curtain and Hans Zwart. An introduction to infinite-dimensional linear systems theory, volume 21 of Texts in Applied Mathematics. Springer-Verlag, New York, 1995.

[13] Neda Darivandi, K. A. Morris, and Amir Khajepour. An algorithm for LQ-optimal actuator location. Smart Materials and Structures, 22(3):035001, 2013.

[14] Pierre Grisvard. Elliptic problems in nonsmooth domains. Classics in applied mathematics. Society for Industrial and Applied Mathematics, 2011.

[15] H. R. Grümm. Two theorems about $\mathscr{C}_{p}$. Reports on Mathematical Physics, 4(3), 1973.

[16] D. Kasinathan and K. A. Morris. $H_{\infty}$-optimal actuator 

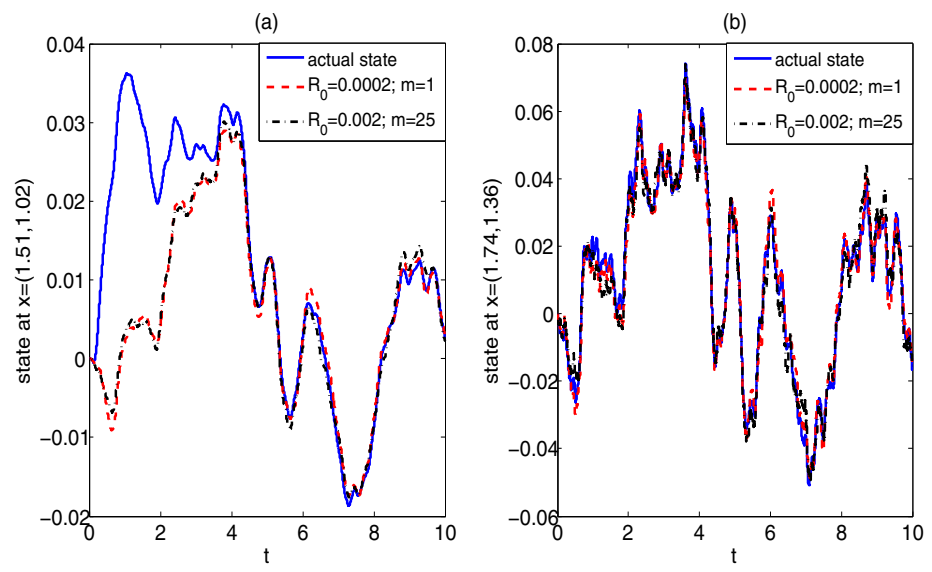

Fig. 16. Comparison of two estimates: one obtained when using a single optimally placed sensor with $r_{0}=0.0002$ (red dashed line) and the other obtained when using 25 optimally placed sensors with $r_{0}=0.002$ (black dotted line), with the actual state (blue solid line) at points (a) $x=(1.51,1.02)$, (b) $x=(1.74,1.36)$.

location. IEEE Tran. on Automatic Control, 58(10):2522 $-2535,2013$.

[17] T. Khan, K. A. Morris, and M. Stastna. Computation of the optimal sensor location for the estimation of a linear dispersive wave equation. In American Control Conference, 2015.

[18] Sarah King, Wei Kang, and Liang Xu. Observability for optimal sensor locations in data assimilation. Int. J. Dyn. Control, 3(4):416-424, 2015.

[19] G. N. Milstein. Numerical integration of stochastic differential equations, volume 313 of Mathematics and its Applications. Kluwer Academic Publishers Group, Dordrecht, 1995. Translated and revised from the 1988 Russian original.

[20] K. A. Morris. Design of finite-dimensional controllers for infinite-dimensional systems by approximation. Jour. of Mathematical Systems, Estimation and Control, 4(2):130, 1994.

[21] K. A. Morris. Linear-quadratic optimal actuator location. IEEE Trans. Automat. Control, 56(1):113-124, 2011.

[22] Yannick Privat, Emmanuel Trélat, and Enrique Zuazua. Optimal observation of the one-dimensional wave equation. Journal of Fourier Analysis and Applications, 19(3):514-544, 2013.

[23] Yannick Privat, Emmanuel Trélat, and Enrique Zuazua. Optimal shape and location of sensors for parabolic equations with random initial data. Archive for Rational Mechanics and Analysis, 216(3):921-981, 2014.

[24] Yannick Privat, Emmanuel Trélat, and Enrique Zuazua. Optimal observability of the multi-dimensional wave and Schrödinger equations in quantum ergodic domains. Journal of the European Mathematical Society, 18(5):1043-1111, 2016.

[25] Martin Weickgenannt, Stefan Neuhaeuser, Benjamin Henke, Werner Sobek, and Oliver Sawodny. Optimal sensor placement for state estimation of a thin doublecurved shell structure. Mechatronics, 23(3):346 - 354,
2013.

[26] Joachim Weidmann. Linear operators in Hilbert spaces, volume 68 of Graduate Texts in Mathematics. SpringerVerlag, New York, 1980. Translated from the German by Joseph Szücs.

[27] X. Wu, B. Jacob, and H. Elbern. Optimal control and observation locations for time-varying systems on a finite-time horizon. SIAM J. Control and Optim., 54(1):291-316, 2015.

[28] S. D. Yang and K. A. Morris. Comparision of actuator placement criteria for control of beam vibrations. J. of Sound and Vibration, 353:1-18, 2015.

[29] Minxin Zhang and Kirsten Morris. Effect of sensor noise on estimation of diffusion. IFAC-PapersOnLine, 49(8):60 - 65, 2016. 2nd IFAC Workshop on Control of Systems Governed by Partial Differential Equations CPDE 2016, Bertinoro, Italy, 13 -15 June, 2016.

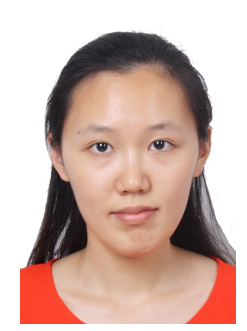

Minxin Zhang received the B.S. degree in Mathematics from Wuhan University, China, in 2014, and the M.Math degree in Applied Mathematics from University of Waterloo, Canada, in 2016. Starting in Fall 2017, she will pursue a Ph.D. degree in Mathematics in University of California, San Diego. Her current research interests include: control of distributed parameter systems, infinite-dimensional Kalman filtering, and computational optimization.

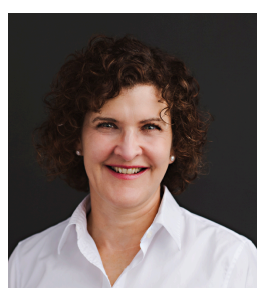

Kirsten Morris 's research interests are systems modelled by partial differential equations and also systems, such as smart materials, involving hysteresis. She has written an undergraduate textbook "Introduction to Feedback Control", and was editor of the book ?Control of Flexible Structures? She received her $\mathrm{PhD}$ in Electrical Engineering from the University of Waterloo and is now a professor in the Applied Mathematics Department at that University with a cross-appointment to the Department of Mechanical \& Mechatronics Engineering. From 2005-2008 she was Associate Dean for Graduate Studies \& Research in the Faculty of Mathematics. Prof. Morris served as a vice-president of the IEEE Control System Society from 2013-2016. She was an associate editor with the IEEE Transactions on Automatic Control and is currently an editor for SIAM Journal on Control \& Optimization, as well as Mathematics of Control, Signals and Systems and is a member of the editorial board of the SIAM book series Advances in Design \& Control. 